\title{
Monitoring protein conformational changes using fluorescent nanoantennas
}

\author{
Scott G. Harroun $\oplus^{1}$, Dominic Lauzon ${ }^{1}$, Maximilian C. C. J. C. Ebert ${ }^{2}$, Arnaud Desrosiers ${ }^{13}$, \\ Xiaomeng Wang ${ }^{1}$ and Alexis Vallée-Bélisle $\mathbb{1}^{1,3 凶}$
}

\begin{abstract}
Understanding the relationship between protein structural dynamics and function is crucial for both basic research and biotechnology. However, methods for studying the fast dynamics of structural changes are limited. Here, we introduce fluorescent nanoantennas as a spectroscopic technique to sense and report protein conformational changes through noncovalent dye-protein interactions. Using experiments and molecular simulations, we detect and characterize five distinct conformational states of intestinal alkaline phosphatase, including the transient enzyme-substrate complex. We also explored the universality of the nanoantenna strategy with another model protein, Protein $\mathbf{G}$ and its interaction with antibodies, and demonstrated a rapid screening strategy to identify efficient nanoantennas. These versatile nanoantennas can be used with diverse dyes to monitor small and large conformational changes, suggesting that they could be used to characterize diverse protein movements or in high-throughput screening applications.
\end{abstract}

E xperimental study of protein transient states ${ }^{1}$ remains a major challenge because high-structural-resolution techniques, including nuclear magnetic resonance and X-ray crystallography, often cannot be directly applied to study short-lived protein states. In contrast, high-temporal-resolution fluorescence spectroscopy is better suited to detect transient states ${ }^{2,3}$, in addition to being convenient, highly sensitive and widely available. While large conformational changes can often be detected using intrinsic tryptophan fluorescence ${ }^{4}$, those in the $\sim 3-9 \mathrm{~nm}$ range are typically detected using Förster resonance energy transfer (FRET). FRET, however, requires the complicated chemistry of site-specific insertion of fluorophores ${ }^{5,6}$. Thus, despite much progress in fluorescence techniques ${ }^{7-10}$, there remains an unmet need for sensitive strategies that are able to detect the small conformational changes ${ }^{11}$ experienced by some proteins during their function, such as enzyme catalysis $^{12}$, as well as for simpler and more efficient labeling strategies.

Here, we report the design, engineering and application of a versatile tool called fluorescent nanoantennas, which sense and report protein conformational change, and in turn protein function, in real time. Since widely available fluorescent dyes display low affinity for proteins ${ }^{13,14}$, these nanoantennas are designed to drive noncovalent dye-protein interactions, making them highly sensitive to conformational changes. Each dye ought to have an affinity for a different region of a protein, depending on their structural complementarity and chemical properties. Thus, via highly programmable phosphoramidite chemistry, we synthesized nucleic acid (DNA) and polyethylene glycol (PEG) nanoantennas containing dyes and other functional modifications. We also leveraged the convenience of biotin-streptavidin (SA) noncovalent interaction, which enables quick and easy connection of biotin-labeled nanoantennas to biotin-labeled proteins.

As a first model protein to test whether nanoantennas can detect protein activity, we selected calf intestinal alkaline phosphatase (AP; EC 3.1.3.1 $)^{15}$. The study of intestinal AP is an active area of research ${ }^{16}$ due to its important roles in preventing inflammation ${ }^{17}$, promoting growth of the commensal microbiota ${ }^{18}$, regulating $\mathrm{pH}^{19}$, activating prodrugs $^{20,21}$ and studies of fundamental biophysics ${ }^{22}$. APs have been implicated in breast, prostate, colorectal and gastric cancers ${ }^{23-26}$, metabolic syndrome ${ }^{27}$, hypophosphatasia ${ }^{28,29}$, myocardial infarction $^{30}$, chronic intestinal inflammation ${ }^{31}$ and even SARS-CoV-2 infection ${ }^{32}$. This enzyme has been characterized by crystallography $^{33-35}$, computational simulations ${ }^{36}$, unfolding ${ }^{37}$, inhibitors ${ }^{38,39}$, mutations $^{40}$ and hydrolysis of substrates ${ }^{40,41}$. Classic ${ }^{42,43}$ and newer strategies $^{4-46}$ to characterize AP-mediated hydrolysis in real time involve monitoring the rate of product generation (Extended Data Fig. 1). Unfortunately, these assays require synthetic substrates to provide a signal, whereas biomolecular substrates of AP are spectroscopically silent (for example, nucleotide triphosphates) ${ }^{18,19}$. For biomolecules, the standard malachite green assay does not permit real-time analysis ${ }^{47}$, while alternative biomolecular assays are not universal $^{48,49}$. Isothermal titration calorimetry ${ }^{50,51}$ can characterize AP activity for biological substrates ${ }^{52}$, but it is not amenable to high-throughput screening. We are not aware of any FRET studies involving labeling of AP, presumably due to the small conformational changes experienced by this protein ${ }^{34,53}$. Here, we designed fluorescent nanoantennas, investigated their signaling mechanism and applied them to study AP function as well as a second protein system, Protein $\mathrm{G}$ interacting with various antibodie ${ }^{54}$.

\section{Results}

Mechanism of fluorescent nanoantennas. We summarize the general idea of our strategy in Fig. 1a. The DNA- or PEG-based fluorescent nanoantennas contain a fluorescent dye at one end, such as fluorescein (FAM), and biotin at the other to facilitate attachment (Start). Using biotin, we attached the nanoantenna to wild-type tetrameric SA from Streptomyces avidinii, which has four biotin-binding sites, and observed a decrease (or quenching) in FAM fluorescence (Step 1). Docking simulations (Fig. 1b), experimental evidence (Extended Data Fig. 2) and previous studies $^{55,56}$ suggest that FAM binds near the unoccupied biotin-binding

'Laboratoire de Biosenseurs \& Nanomachines, Département de chimie, Université de Montréal, Montréal, Québec, Canada. ${ }^{2} \mathrm{Chemical}$ Computing Group ULC, Montréal, Québec, Canada. ${ }^{3}$ Département de biochimie et médecine moléculaire, Université de Montréal, Montréal, Québec, Canada. ${ }^{凶}$ e-mail: 
a
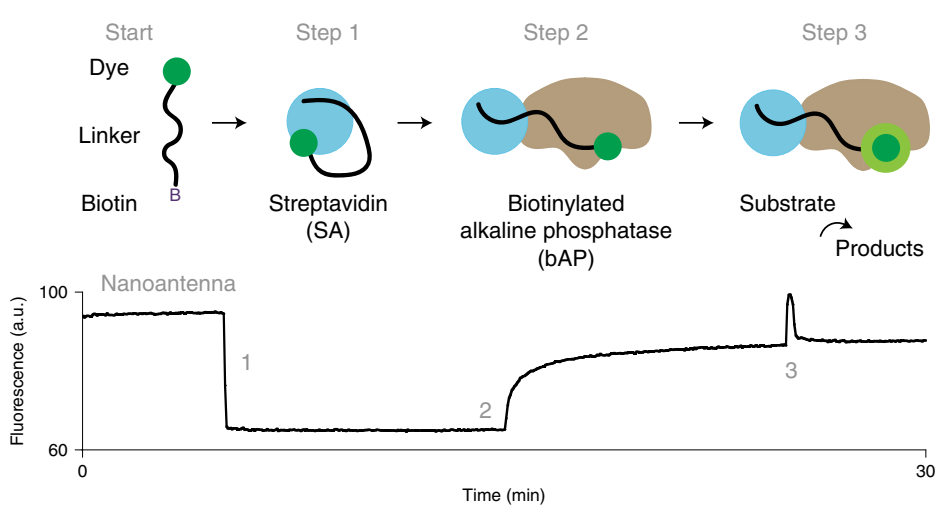

b
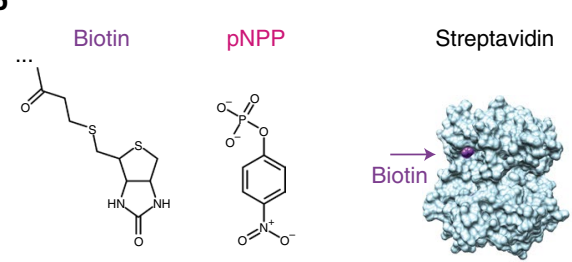

c

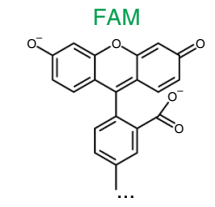<smiles>[CH2-]C(=O)c1ccccc1-c1c2cc(C)c(=NCC)cc-2oc2cc(C)c(NCC)cc12</smiles>

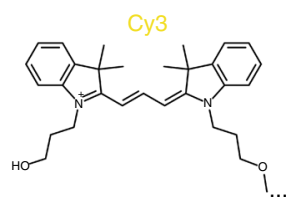

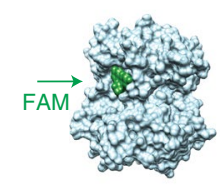
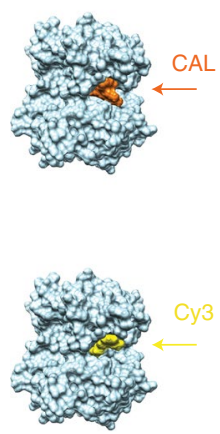
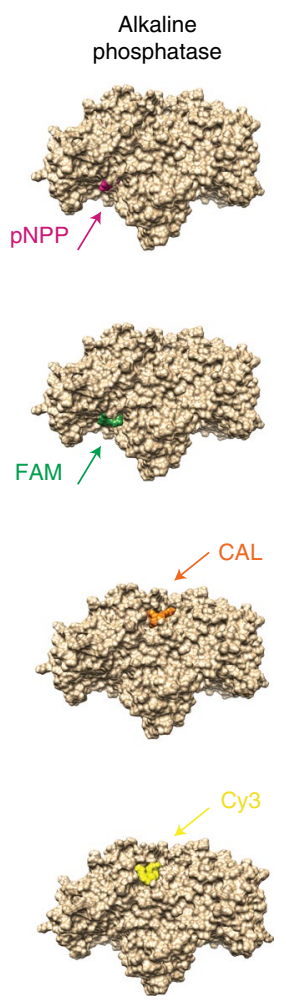
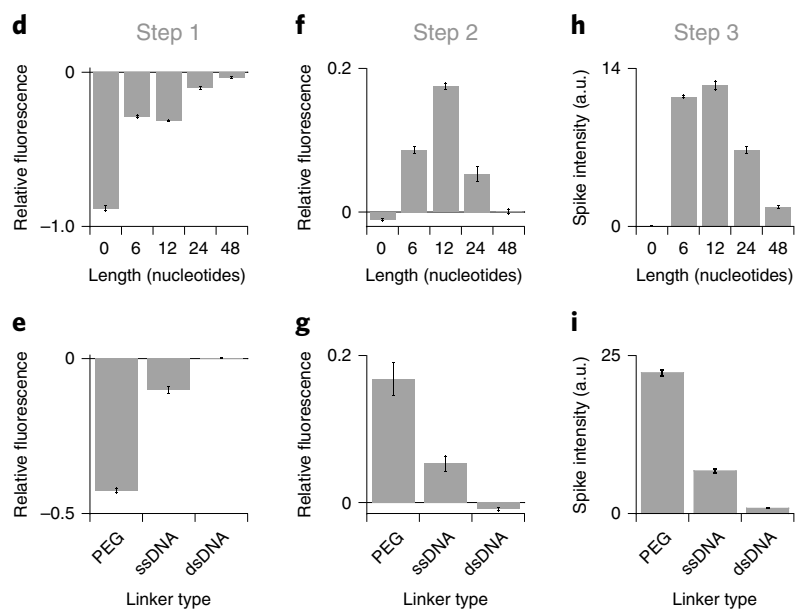

i

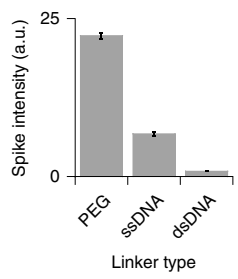

j

m

n

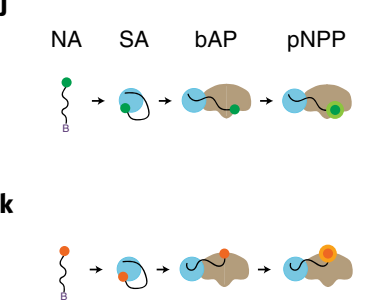

I

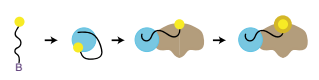

CDNA
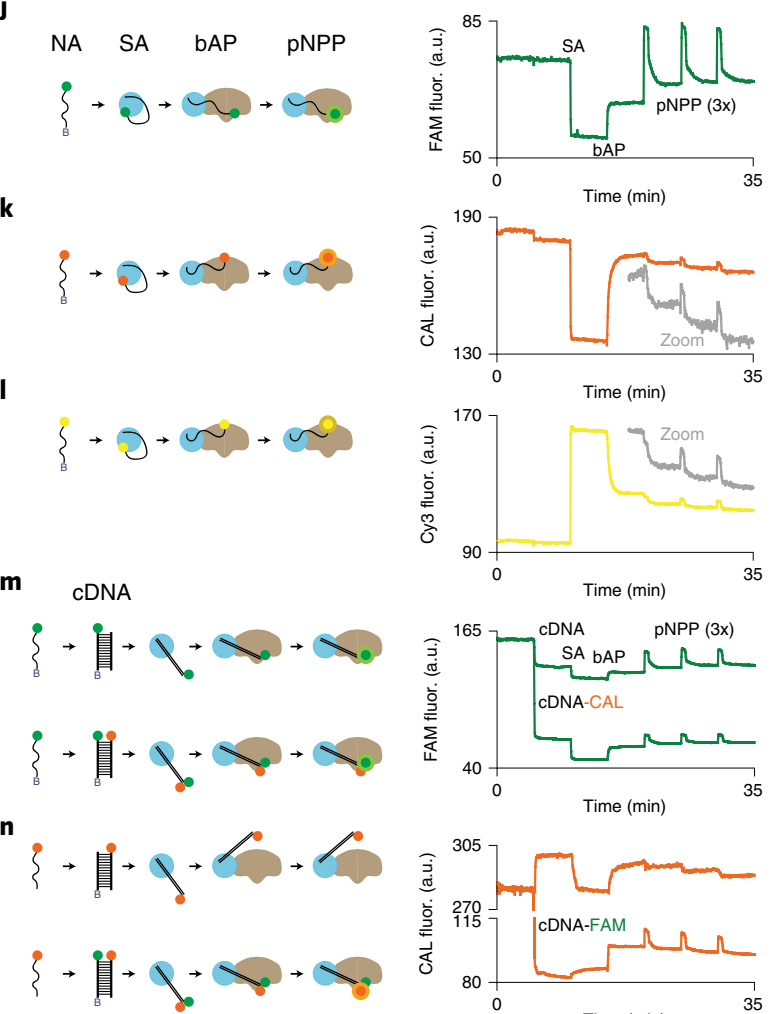

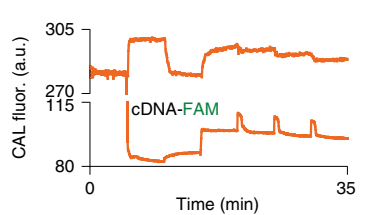

Fig. 1 | Overview of the fluorescent nanoantenna strategy to probe different regions on a protein. $\mathbf{a}$, Cartoon and example data of fluorescent nanoantennas. For simplicity, the cartoon shows only one of each component. a.u., arbitrary units. b, The docking simulation accurately predicts the binding sites of biotin on SA and the substrate pNPP on AP. c, Docking prediction of the dyes FAM, CAL and Cy3 on SA and AP. $\mathbf{d}-\mathbf{i}$, Optimization of the length (d) and composition (e) of the linker for Step 1 and similarly for Step 2 (length, f; composition, g). See Supplementary Fig. 1 for corresponding fluorescence spectra. Similar results (length, $\mathbf{h}$; composition, i) were observed for the fluorescence spike during hydrolysis of pNPP for Step 3.

For $\mathbf{d}-\mathbf{i}, n=1$ biologically independent enzyme samples were examined over three independent experiments. Data are presented as mean values \pm s.e.m. $\mathbf{j}-\mathbf{I}$, Kinetic signatures of ssDNA nanoantennas (NA) containing the dye FAM $\left(\lambda_{\text {ex }}=498 \mathrm{~nm}, \lambda_{\text {em }}=520 \mathrm{~nm}\right)(\mathbf{j}), C A L\left(\lambda_{\text {ex }}=540 \mathrm{~nm}, \lambda_{\text {em }}=561 \mathrm{~nm}\right)(\mathbf{k})$ or Cy3 $\left(\lambda_{\text {ex }}=546 \mathrm{~nm}, \lambda_{\text {em }}=563 \mathrm{~nm}\right.$ ) (I) for SA and bAP binding events, as well as pNPP hydrolysis. fluor., fluorescence. $\mathbf{m}, \mathbf{n}$, Double-dye competition kinetic signatures for FAM (m) and CAL (n). In $\mathbf{m}$, the data at the top show the monitoring of FAM fluorescence of a single-dye dsDNA nanoantenna with FAM, and the data at the bottom show the monitoring of FAM fluorescence of a double-dye dsDNA nanoantenna with FAM and CAL. In $\mathbf{n}$, the data at the top show the monitoring of the CAL fluorescence of a single-dye dsDNA nanoantenna with $C A L$, and the data at the bottom show the monitoring of the CAL fluorescence of a double-dye dsDNA nanoantenna with FAM and CAL.

sites of SA. Next, we added the model protein, biotinylated calf intestinal alkaline phosphatase (bAP). Unlike specific dye labeling required for FRET experiments, our method employed nonspecific biotinylation, which can be performed conveniently on many proteins without affecting their function ${ }^{57}$. Binding of bAP to the
nanoantenna-SA platform results in an increase in the fluorescence signal (Step 2), suggesting that FAM is released from SA. Upon addition of a substrate of AP, the nanoantenna generates a transient fluorescence 'spike' (Step 3), enabling real-time monitoring of the enzyme's transient substrate-bound state. This result, combined 

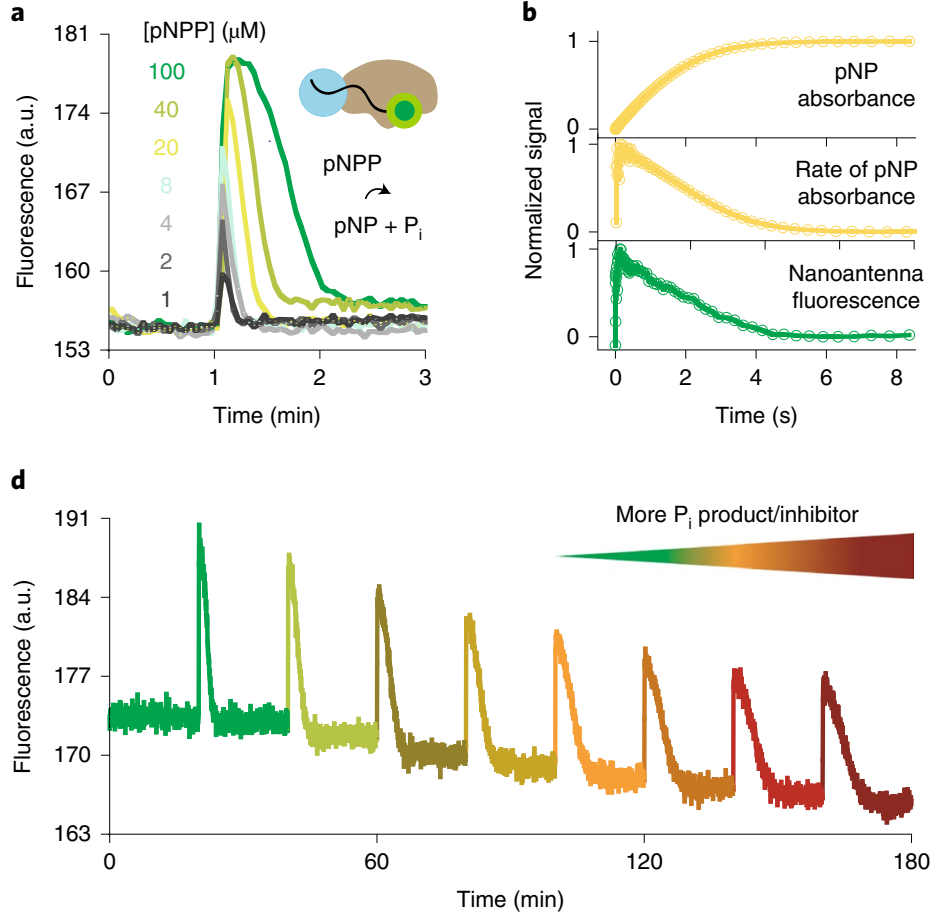
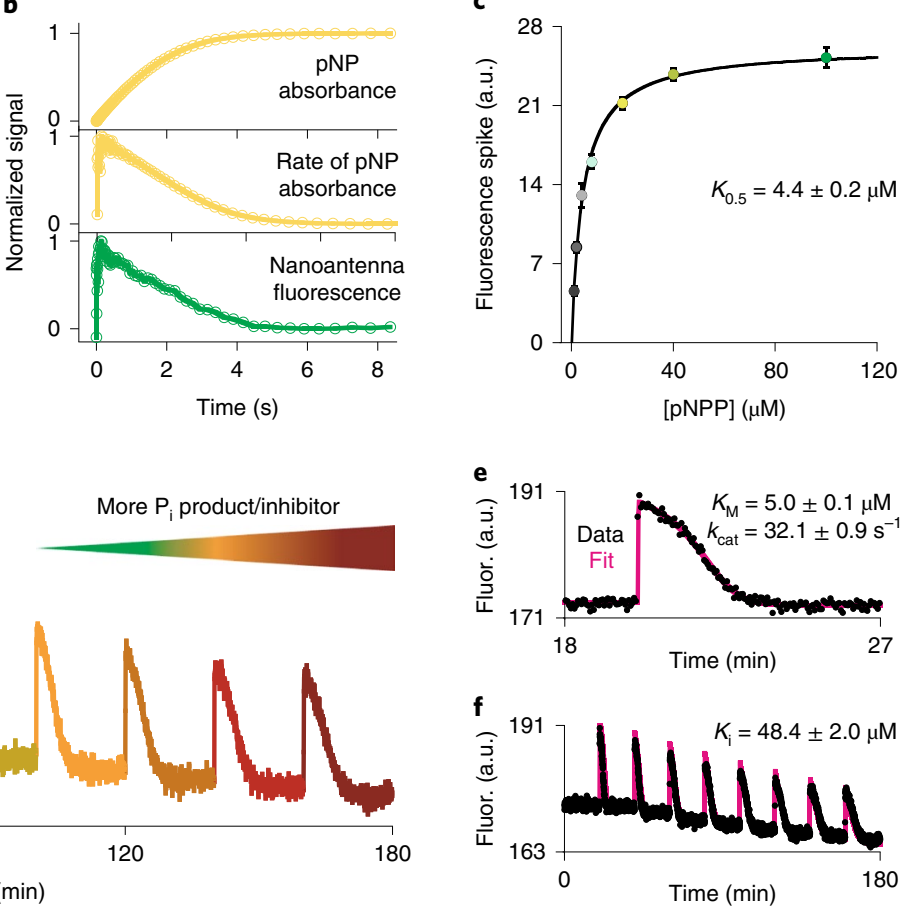

Fig. 2 | Fluorescent nanoantennas enable complete characterization of the enzyme's kinetic mechanism. a, Increasing the substrate concentration increases the fluorescence spike intensity and duration, displaying a profile reminiscent of the enzyme-substrate concentration ([ES]) during a typical enzymatic reaction. $\mathbf{b}$, Fluorescence intensity is correlated with the rate of reaction (and [ES]) determined by monitoring pNP generation via UV-visible spectroscopy. c, The saturation binding curve realized using the nanoantenna spike intensity displays a typical Michaelis-Menten-like plot. d, Nanoantenna-labeled enzyme allows sequential injection of pNPP without signal saturation. Here, subsequent pNPP injection ( $30 \mu M$ ) results in prolonged reaction time and reduced spike intensity due to competitive inhibition via the accumulation of $\mathrm{P}_{\mathrm{i}}$. $\mathbf{e}$, Extracting $K_{\mathrm{M}}$ and $k_{\text {cat }}$ using the fluorescent nanoantenna signature of a single reaction (see Methods, Supplementary Fig. 25 and 'Script for fitting kinetic data in MATLAB' in the Supplementary Information for details of the fitting procedure). $\mathbf{f}$, Extracting $K_{\mathrm{i}}$ using the fluorescent nanoantenna signature of multiple reactions. All experiments were performed with $n=1$ biologically independent enzyme samples examined over three independent experiments. Data are presented as mean values \pm s.e.m. In a-c, we used $100 \mathrm{nM}$ bAP from a commercially available sample, and in $\mathbf{d}-\mathbf{f}$ we used $10 \mathrm{nM}$ bAP prepared by us from AP and a biotinylation kit.

with docking simulations (Fig. 1c), suggests that FAM binds near one of the two equivalent active sites.

We explored how the nanoantenna linker length (LX, where X is the number of nucleotides) (Fig. 1d) and composition (Fig. 1e) impact dye-protein interactions. As a 'no linker' L0 nanoantenna, we selected a biotin-fluorescein conjugate. Upon binding to SA, its fluorescein moiety is located just outside the biotin-binding site that it occupies ${ }^{58}$. This short nanoantenna displayed substantial fluorescence quenching. Using single-stranded DNA (ssDNA), we increased the linker length to L6 or L12, thereby enabling FAM to interact with more of the SA surface. These nanoantennas displayed moderate quenching, with their FAMs likely binding near unoccupied biotin-binding sites (Fig. 1b) ${ }^{55,56}$. The longer L24 and L48 nanoantennas displayed reduced quenching, consistent with fewer dyes being bound to the protein due to the lower effective concentration of the dye near SA. We also tried a more flexible, hydrophilic and less charged PEG-based nanoantenna (approximately L21, Supplementary Fig. 2), which displayed increased quenching. In contrast, a less flexible double-stranded DNA (dsDNA) L24 nanoantenna prevented the FAM-SA interaction.

Linker length (Fig. 1f) and composition (Fig. 1g) likewise affected the monitoring of protein binding to SA. As expected, due to its short length, L0 did not detect bAP. The longer L6 and especially L12 nanoantennas enabled FAM to detect bAP attachment, but L24 and especially L48 were too long to result in a high local concentration of FAM near bAP. Also as expected, we observed that a PEG linker enabled good FAM-bAP interaction, while a less flexible
dsDNA linker did not. A molecular dynamics (MD) simulation with the optimal L12 nanoantenna revealed that its FAM could plausibly reach the bound bAP, supporting our hypothesis regarding the dye-enzyme interaction (Extended Data Fig. 3 and Supplementary Video 1). We have additionally explored other factors, such as $\mathrm{pH}$ variation and the ratio of components, and found that PEG linkers were less sensitive to $\mathrm{pH}$ variation (Supplementary Fig. 3), while using too many nanoantennas per streptavidin diminishes signaling by preventing enzyme attachment (Supplementary Figs. 4-6).

We next investigated the mechanism by which the nanoantennas generated a transient fluorescence spike during hydrolysis of p-nitrophenylphosphate (pNPP; step 3 in Fig. 1a). As expected, the most sensitive nanoantennas for probing bAP attachment were also the most sensitive for probing its catalytic activity (Fig. 1h,i and Supplementary Fig. 7). The stoichiometry of the added components is important; we found that adding three nanoantennas per SA maximizes signal without potentially limiting the ability of the biotinylated enzyme to bind to the remaining unoccupied biotin-binding site(s). Nanoantenna size, and therefore steric hindrance $^{59}$, is also a factor (Supplementary Fig. 7d). As controls, we noted that no spike occurred when there was no hydrolysis reaction, for example, upon addition of the reaction products (Extended Data Fig. $4 \mathrm{a}-\mathrm{c}$ ) or upon addition of pNPP when unattached nonbiotinylated nanoantennas were employed (Extended Data Fig. 4d). We also did not observe a signal change due to intrinsic tryptophan fluorescence (Supplementary Fig. 8) ${ }^{4}$, and could not detect AP function with the protein-binding dye 8-anilinonaphthalene-1-sulfonic 

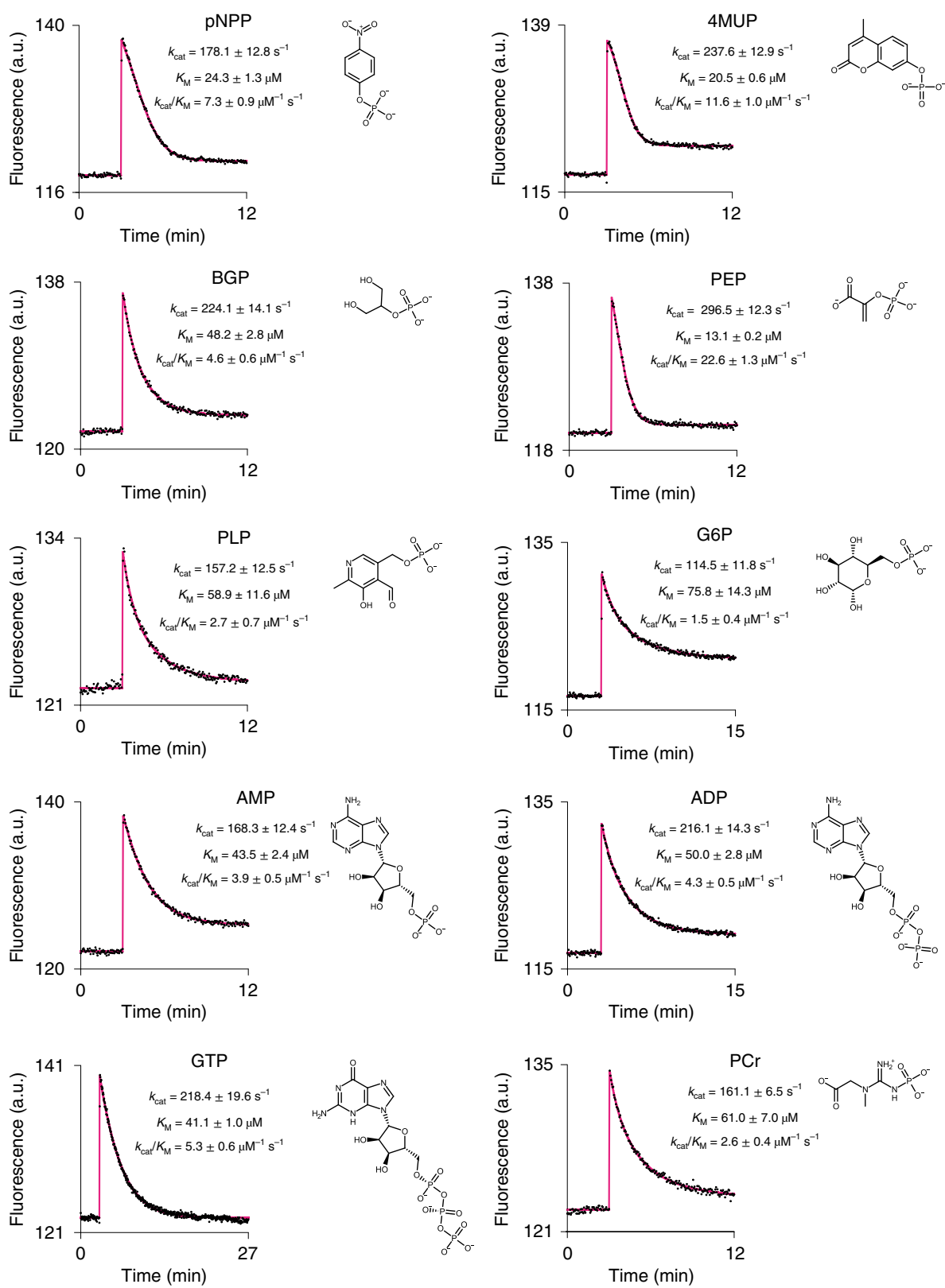
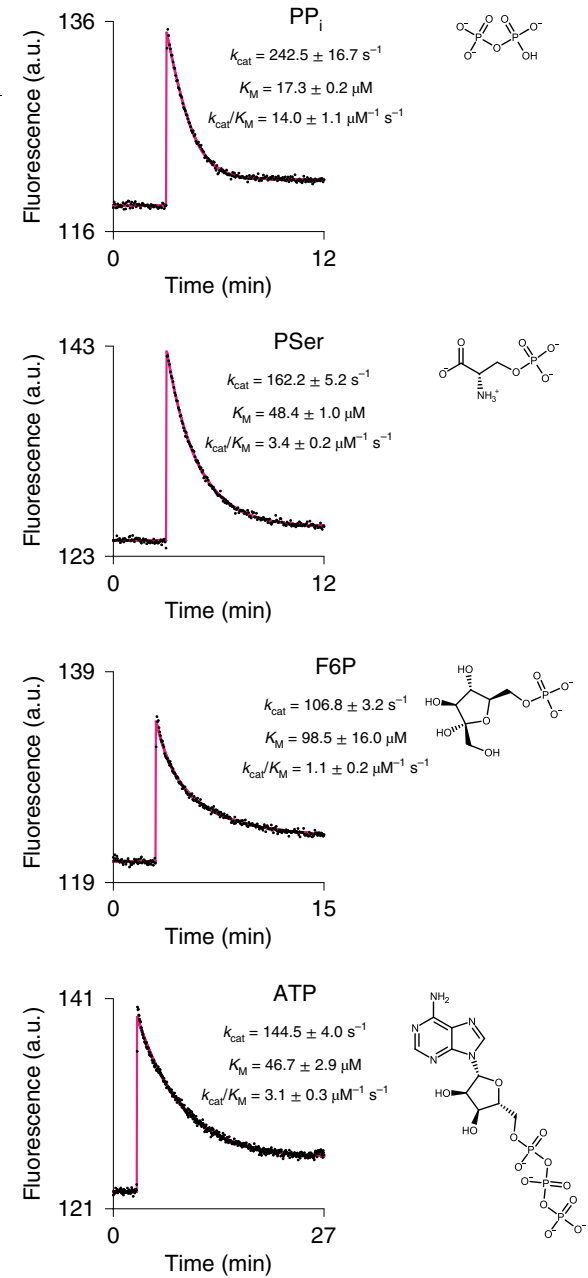

Fig. 3 | Fluorescent nanoantennas enabled real-time monitoring of any substrate hydrolyzed by AP. Nanoantenna fluorescence signatures during hydrolysis of pNPP, 4MUP, PP ${ }_{i}$, BGP, PEP, PSer, PLP, G6P, F6P, AMP, ADP, ATP, GTP, PCr and amifostine (all $300 \mu M$ ). All experiments were performed with $n=1$ biologically independent enzyme samples examined over three independent experiments. Data are presented as mean values \pm s.e.m.

acid (Supplementary Fig. 9) ${ }^{60}$. We did, however, observe a spike with other nanoantenna attachment strategies (for example, covalent attachment to surface-exposed lysine residues of AP; Extended Data Fig. 4e-h), different buffer conditions (Supplementary Fig. 10) and various storage times (Supplementary Fig. 11). We also observed that the intensity of the nanoantenna's fluorescence in the different states (that is, after addition of SA, bAP and pNPP) was sensitive to small chemical modifications that could subtly perturb the FAM-protein interaction, such as a nearby hydrophobic $\mathrm{C}_{16}$ alkane chain (Extended Data Fig. 5) and different chemical connections of FAM to the linker (Extended Data Fig. 6). Finally, under some conditions, we observed that the fluorescence spike intensity during pNPP hydrolysis was greater than the initial fluorescence of the unbound nanoantenna (Fig. $1 \mathrm{j}$ and Supplementary Fig. 12). FAM simply being ejected from the active site by the incoming pNPP substrate would likely just return the fluorescence to the initial baseline before protein binding. Overall, these results reinforce our proposed mechanism that the nanoantenna-mediated dye-enzyme interaction enables monitoring of the conformational changes on the enzyme's surface during its function.

The testing of chemically diverse dyes on ssDNA L12 nanoantennas provides information about the signaling mechanism and potential universality of the strategy. Different dyes are predicted to bind to different sites on AP (Fig. 1c and Supplementary Figs. 13 and 14). Interestingly, all nine dyes tested enabled monitoring of the SA and bAP binding events, albeit weakly in some cases. Shown here are FAM, the rhodamine-based CAL Fluor Orange 560 (CAL) and cyanine 3 (Cy3) (Fig. 1j-1; see other dyes in Supplementary Fig. $15 \mathrm{a}-\mathrm{i})$. In contrast to FAM, Cy3 shows increased fluorescence upon binding to $\mathrm{SA}$ and decreased fluorescence upon binding to $\mathrm{bAP}^{7}$, and it is more sensitive with a dsDNA linker. However, similar to FAM, it is affected by its chemical connection (Supplementary Fig. 15e,f). 

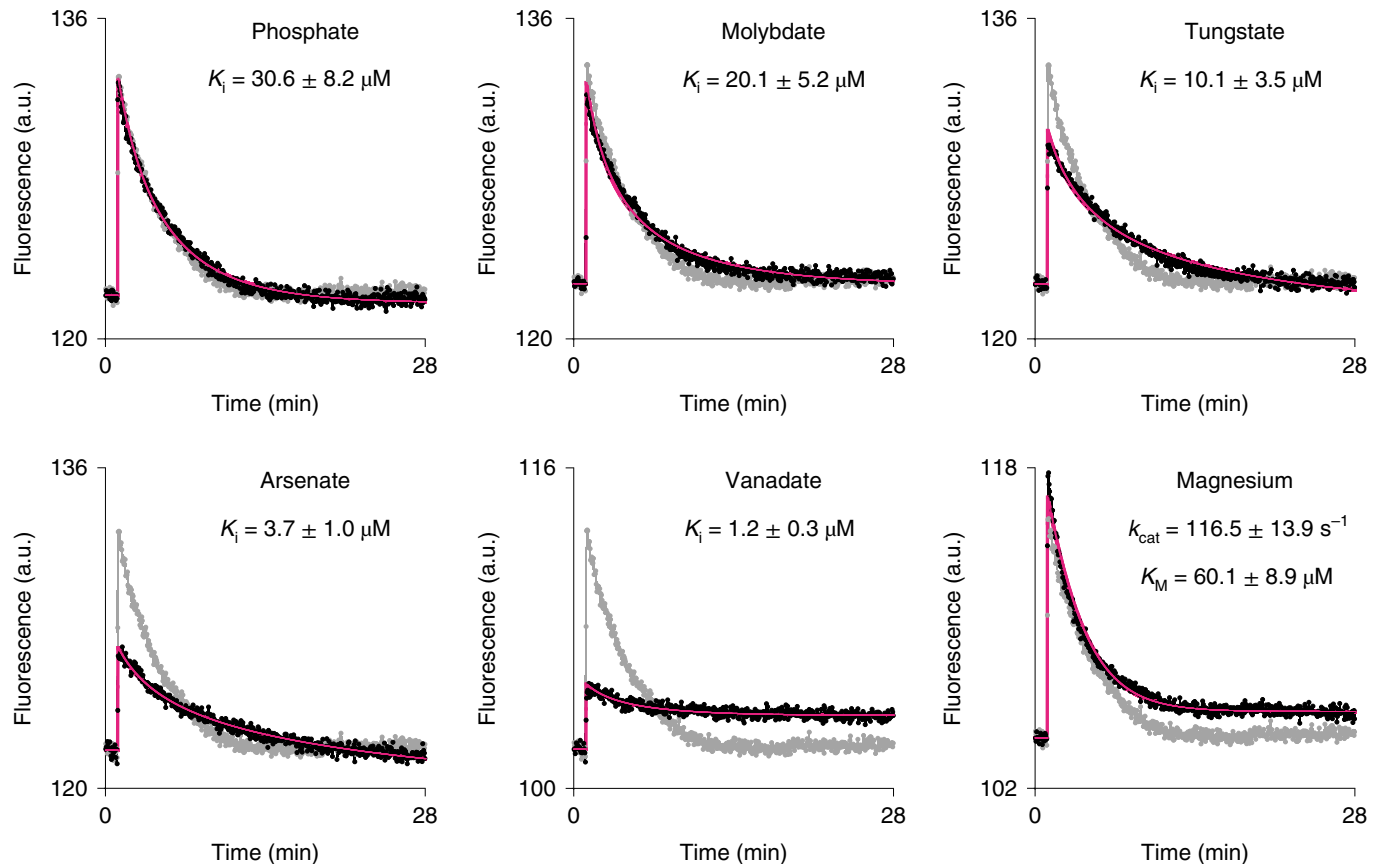

Fig. 4 | Screening inhibitors by monitoring AP hydrolysis using fluorescent nanoantennas. The inhibitory effects on AP of phosphate, molybdate, tungstate, arsenate and vanadate, as well as the effect of $\mathrm{Mg}^{2+}$ ion (black) with the substrate amifostine. Kinetic fitting of the data is overlaid (pink). Shown also for comparison is the hydrolysis of amifostine without inhibitor (gray). The baseline of the kinetic signature without the effector was adjusted for presentation of data (that is, due to the fluorescence quenching caused by vanadate and magnesium ion). All experiments were performed with $n=1$ biologically independent enzyme samples examined over three independent experiments. Data are presented as mean values \pm s.e.m. In all experiments, we used $30 \mu \mathrm{M}$ inhibitor or $5 \mathrm{mM} \mathrm{Mg}^{2+}$, and otherwise the same conditions as in Fig. 3.

We also found that the kinetics of the signal change upon addition of bAP depend both on the concentration of bAP (Supplementary Fig. 16) and on the nanoantenna properties (Supplementary Fig. 17). This suggests that dye dissociation from SA is not rate limiting and that the dye-binding location and the nature of the linker may affect the rate at which bAP binds to SA (for example, through steric hindrance). We further observed that nanoantennas with FAM, CAL and Cy3 enabled monitoring of pNPP hydrolysis. Crucially, while the three dyes provided different sensitivities toward the functional events, they all exhibited the same kinetics for pNPP hydrolysis (Supplementary Fig. 15j), indicating that the dye-protein interactions exploited herein did not interfere with protein function. From a practical perspective, FAM remains the best dye for monitoring AP function. However, from a mechanistic perspective, the other dyes provide strong evidence that nanoantennas employing chemically diverse dyes can be used to probe conformational changes at different locations.

We investigated the signaling mechanisms of dyes predicted to bind at different locations. For nanoantennas with FAM, we proposed three mechanisms for modulation of fluorescence during AP function: (1) binding of pNPP at the active site directly ejects FAM from its nearby binding site; (2) small conformational changes alter FAM's affinity for AP and release it from the binding site; or (3) small conformational changes perturb the emission of the bound FAM. For CAL and $\mathrm{Cy} 3$, which are predicted to bind at locations distal to the active site, we proposed only the latter two mechanisms. Thus we used MD simulations and examined the trajectories of the bound dyes in the presence or absence of bound pNPP (Extended Data Fig. 7). FAM remained bound near the active site both in the absence and in the presence of pNPP, suggesting a strong affinity for this site. In contrast, CAL was not stabilized in its initial binding site in either the absence or presence of pNPP, suggesting low affinity. Interestingly, Cy3 remained bound in the absence of pNPP but dissociated in its presence. These simulations, therefore, suggest a FAM-signaling mechanism that is not based on ejection by pNPP nor a change in affinity, but instead by the sensing of small conformational changes in the local chemical environment ${ }^{61,62}$. For $\mathrm{CAL}$, the mechanism remains uncertain, but for $\mathrm{Cy} 3$, the simulations suggest that conformational changes during pNPP hydrolysis transiently release the dye. The bound and unbound states of Cy3 could, for example, affect its cis-trans isomerism and therefore its fluorescence $\mathrm{e}^{7,63,64}$.

If the sensitivity of a nanoantenna truly depends on whether the dye's binding location experiences conformational change during protein function, one ought to observe a change in the fluorescence signature upon forcing the dye to bind at another location. To test this hypothesis, we employed a dsDNA L12 nanoantenna containing both FAM and CAL (Fig. $1 \mathrm{~m}, \mathrm{n}$ ). Our reasoning was that the two dyes will compete for their preferred binding sites on bAP. On the basis of the above MD simulations, we expected that the higher affinity FAM will bring the lower affinity CAL along with it. Aside from the decrease of signal intensity, likely due to a contact-mediated quenching mechanism between the dyes ${ }^{65}$ (Extended Data Fig. 8), we observed that the presence of CAL does not substantially affect the FAM fluorescent signature (Fig. $1 \mathrm{~m}$ ). In contrast, the addition of FAM substantially affects the CAL fluorescent signature (Fig. 1n). Notably, it now enables CAL to efficiently detect the conformational change of the protein during pNPP hydrolysis (Fig. 1n). Given that CAL likely stacks on FAM (Extended Data Fig. 8), it is plausible that the fluorescent change of CAL is triggered by the same conformational change affecting the FAM dye. Importantly, the presence of the second dye did not affect the kinetics of the substrate hydrolysis (Supplementary Fig. 18a). We also found that employing FAM with other dyes led to similar results (Supplementary Fig. 18b,c). Of note, these changes in the fluorescence signature do not arise due to FAM emitting a signal that overlaps with the 
$\mathbf{a}$
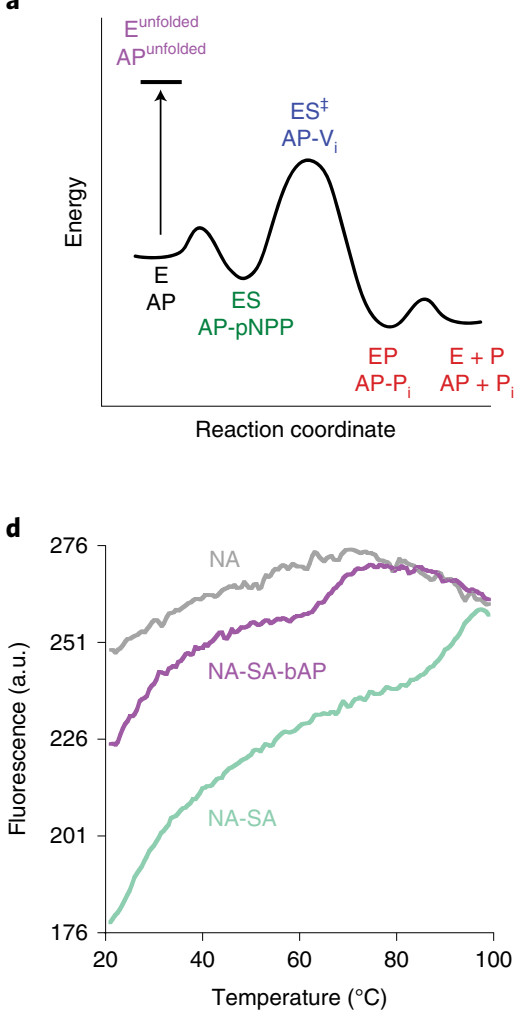

$b$

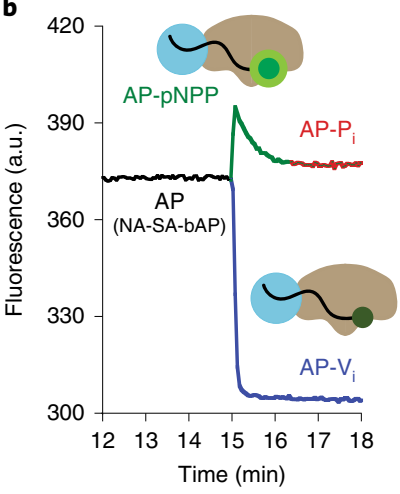

e

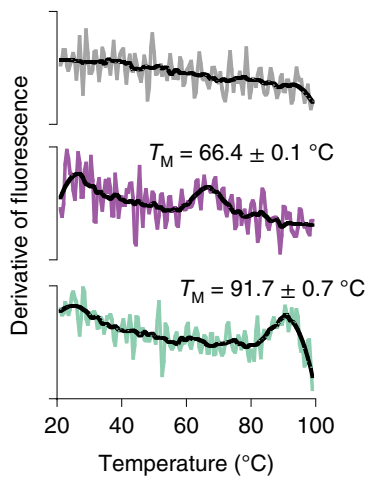

c

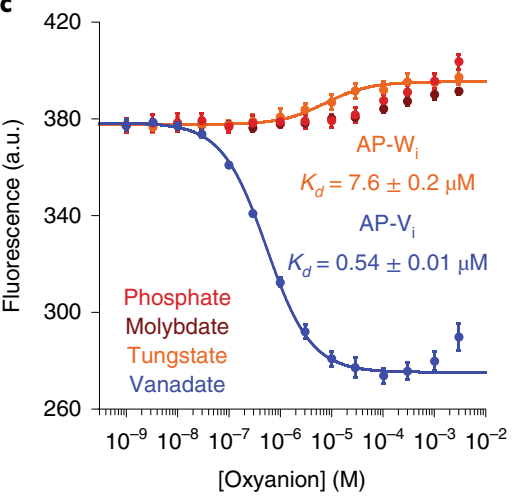

\{

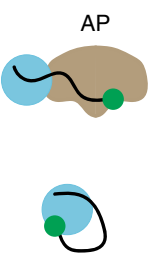

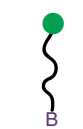

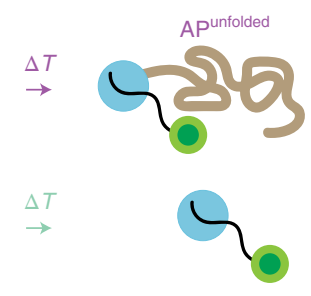

Fig. 5 | Detecting other states of AP using fluorescent nanoantennas. a, Five distinct states of AP detected during enzymatic reaction and denaturation. First, the enzyme (E) is present in solution, followed by the energetically favorable binding of a substrate (for example, pNPP) to form an ES complex. Next, the high-energy transition state (ES) is formed. This state cannot be observed directly but can be studied via TSA inhibitors that mimic its geometry, such as $V_{i}$. Since $V_{i}$ is a competitive inhibitor, it also represents an enzyme-inhibitor complex (not shown). After the hydrolysis of pNPP, the bound $P_{i}$ product $(E P)$ is released from the enzyme $(E+P)$. As a competitive inhibitor, $P_{i}$ can rebind to the active site. AP can also be unfolded by thermal denaturation (Eunfolded). b, Unlike the spike during pNPP hydrolysis, $V_{i}$ binding quenches the nanoantenna's fluorescence. $\mathbf{c}$, Binding curve of the nanoantenna-SA-bAP complex sensing $V_{i}$ (blue) and $W_{i}$ (orange). Other competitive inhibitor oxyanions do not exhibit a substantial fluorescence change. d, Thermal shift assay of the nanoantenna (gray), nanoantenna-SA platform (light green) and nanoantenna-SA-bAP complex (violet). e, Smoothing of the derivatives (black lines) reveals no transition for the unbound nanoantennas, but does for the nanoantenna-SA platform and nanoantenna-SA-bAP complex. In c-e, all experiments were performed with $n=1$ biologically independent enzyme samples examined over three independent experiments. Data are presented as mean values \pm s.e.m.

excitation wavelengths of other dyes (Supplementary Fig. 18d,e). Thus, these experiments provide strong evidence that FAM can redirect other dyes to a location proximal to its own binding site on AP. This observation is consistent with the aforementioned MD simulations, suggesting that FAM is more tightly bound than Cy3 and CAL to AP (Extended Data Fig. 7). Hypothetically, one could rationally employ other non-dye 'molecular anchors' to redirect dyes to specific locations on proteins, as FAM does for CAL on AP.

Characterizing enzyme kinetics. Nanoantennas can be used to characterize the kinetic mechanisms of enzymes. By employing ssDNA L12 FAM nanoantennas hereafter, we first observed that the addition of more pNPP increased the spike intensity and duration (Fig. 2a). The resulting fluorescence signature is reminiscent of the expected profile of the enzyme-substrate concentration ([ES]) during a typical enzymatic reaction (Extended Data Fig. 1b). The signal rapidly peaked and then maintained a steady state until the substrate began to run out. This hypothesis is consistent with the nanoantennas distinguishing between the enzyme and enzyme-substrate conformations. We confirmed the link between the fluorescence intensity of the spike and [ES] by showing that the former is proportional to the rate of reaction obtained by monitoring $p$-nitrophenol (pNP) generation via UV-visible spectroscopy (Fig. 2b). Indeed, plotting the spike intensity versus $\mathrm{pNPP}$ concentration generated a saturation binding curve that is reminiscent of a Michaelis-Menten plot (Fig. 2c; Supplementary Fig. 19). Fitting the data provided a $K_{0.5}$ value that is similar to the Michaelis constant $\left(K_{\mathrm{M}}\right)$ reported in the literature under the same conditions $\left(K_{0.5}=4.4 \pm 0.2 \mu \mathrm{M}\right.$; Supplementary Fig. 20$)^{52}$.

Nanoantennas enable complete kinetic characterization of an enzyme in a single experiment. Unlike the monitoring of AP kinetics by product generation ${ }^{42-44}$, one enzyme sample with nanoantennas can enable multiple measurements in one cuvette. This could be used to characterize various substrates or inhibitors (Fig. $2 \mathrm{~d}$ and Supplementary Fig. 21). For example, upon performing consecutive pNPP injections, we observed a decrease in spike intensity and an increase in reaction time, consistent with accumulation of the product, inorganic phosphate $\left(\mathrm{P}_{\mathrm{i}}\right)$, a competitive inhibitor of this enzyme. We show that by fitting a single fluorescence spike using Michaelis-Menten differential equations with competitive product inhibition $^{51}$, one can extract the $K_{\mathrm{M}}$, the catalytic rate constant $\left(k_{\text {cat }}\right)$ and, from these, the catalytic efficiency $\left(k_{\text {cat }} / K_{\mathrm{M}}\right)$ (Fig. 2e). Indeed, we determined $K_{\mathrm{M}}(5.0 \pm 0.1 \mu \mathrm{M}), k_{\text {cat }}\left(32.1 \pm 0.9 \mathrm{~s}^{-1}\right)$ and $k_{\text {cat }} / K_{\mathrm{M}}$ $\left(6.4 \pm 0.3 \mu \mathrm{M}^{-1} \mathrm{~s}^{-1}\right)$ values similar to those reported in the literature $^{52}$ (Supplementary Table 1 ). Notably, the $K_{\mathrm{M}}$ values determined by plotting spike intensity and by fitting a single spike were also 
a

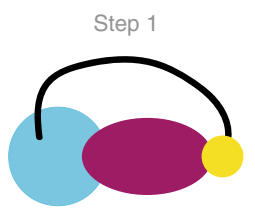

Load platform: biotinylated protein $\mathrm{G}$ (bPG)

b Initial fluorescence

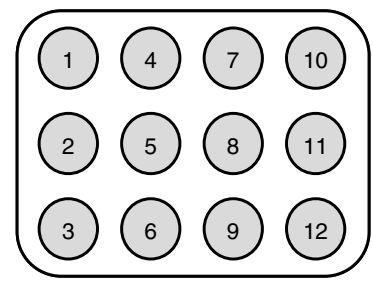

Step 2

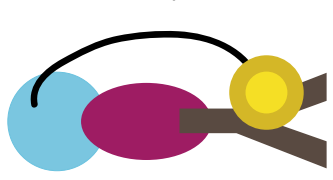

Screen for protein function: binding of goat IgG

Change of fluorescence

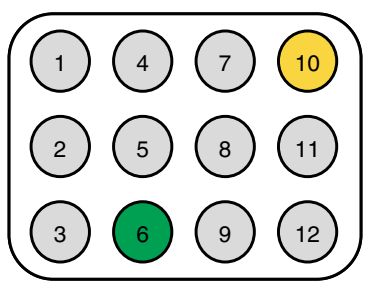

d

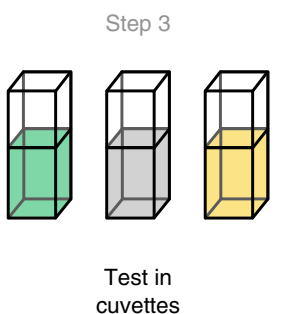

e

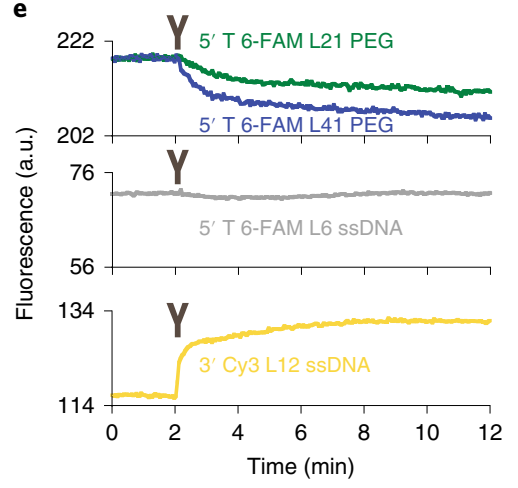

f

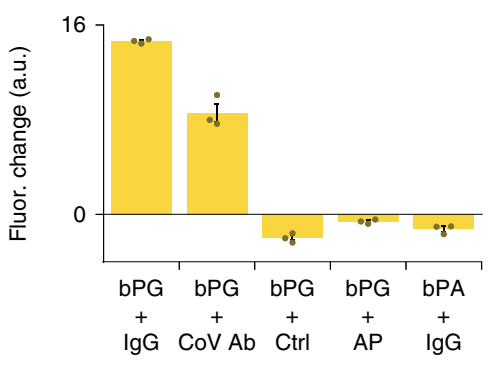

Fig. 6 | Rapid screening strategy for identification of functional nanoantennas for protein $\mathbf{G}$ binding lgG. a,b, Plate reader screening strategy (a) to rapidly identify a nanoantenna that reports the binding of goat IgG to biotinylated protein G (bPG) (b) using the nanoantenna-SA platform. c, Results obtained for rapid screening of 12 nanoantennas for the aforementioned system. $\mathbf{d}$,e, Cuvette validation (d) of results for nanoantennas 1,6 and 10 displays similar trends to the plate reader format. Also shown is the semirationally selected longer FAM L41 PEG (e). f, The nanoantenna-SA-bPG complex detects goat lgG and SARS-CoV-2 lgG/lgM while remaining silent to a sample without SARS-CoV-2 lgG/lgM and to the enzyme AP. As an additional control, replacing bPG with biotinylated protein A (bPA), which does not bind goat IgG, does not display a signal change. Ab, antibody. In c,f, all experiments were performed with $n=1$ biologically independent protein and antibody samples examined over three independent experiments. Data are presented as mean values \pm s.e.m.

consistent. Crucially, similar values were also obtained by monitoring pNP product generation using UV-visible spectroscopy, supporting that neither the nanoantenna nor SA affect the kinetic parameters of the enzyme (Supplementary Figs. 22-24 and Supplementary Table 1). Next, using a similar fitting procedure ${ }^{51}$, we modeled the eight spikes from the same enzyme sample and determined the inhibition constant $\left(K_{\mathrm{i}}\right)$, a measure of the inhibitory effect of the $\mathrm{P}_{\mathrm{i}}$ product (Fig. 2f; see Supplementary Fig. 25a-d for complete fitting example). This $K_{\mathrm{i}}(48.4 \pm 2.0 \mu \mathrm{M})$ was close to the previously reported values (Supplementary Table 2$)^{48,52}$. Moreover, the decrease in spike intensities was consistent with the expected decrease in the reaction rate due to inhibition (Supplementary Fig. 25e).

Fluorescent nanoantennas can be used to monitor the AP-mediated hydrolysis of any substrate, including biomolecules. Indeed, all of the chemically diverse substrates tested herein exhibited a similar fluorescence spike during hydrolysis: pNPP, 4-methylumbelliferylphosphate (4MUP), pyrophosphate
$\left(\mathrm{PP}_{\mathrm{i}}\right)^{29}, \quad \beta$-glycerophosphate $(\mathrm{BGP}), \quad$ phosphoenolpyruvate (PEP), L-phosphoserine (PSer), pyridoxal 5' -phosphate (PLP) ${ }^{29}$, D-glucose-6-phosphate (G6P), D-fructose-6-phosphate (F6P), adenosine $5^{\prime}$-monophosphate (AMP), adenosine $5^{\prime}$-diphosphate (ADP), adenosine $\quad 5^{\prime}$-triphosphate $(\mathrm{ATP})^{19}$, guanosine $5^{\prime}$-triphosphate $(\mathrm{GTP})^{18,19}$, phosphocreatine (PCr) and amifostine ${ }^{20}$ (Fig. 3). Using the 'one-shot' fitting strategy described above, each substrate displayed $K_{\mathrm{M}}$ and $k_{\text {cat }}$ values consistent with previously reported values, when available, while others were characterized with calf intestinal AP for the first time (Fig. 3 and Supplementary Table 3). Furthermore, with 4MUP, which generates fluorescent 4-methylumbelliferone (4MU; also called hymecromone), we found that a single fluorescence spike provided $K_{\mathrm{M}}$ and $k_{\text {cat }}$ values that agreed with those determined by the traditional product generation method (Extended Data Fig. 9). Simply fitting one 4MU product progress curve, however, provided markedly overestimated values ${ }^{66,67}$. In addition to the biomolecular substrates, we also tested amifostine, a prodrug used to protect normal cells during 
chemotherapy and radiotherapy, which is putatively hydrolyzed to its active metabolite form by intestinal $\mathrm{AP}^{20,21}$. While the $K_{\mathrm{M}}$ of amifostine was comparable to that of the other tested substrates, its $k_{\text {cat }}$ was the slowest, likely due to AP not having evolved to process its $\mathrm{P}-\mathrm{S}$ bond, unlike for the $\mathrm{P}-\mathrm{O}$ and $\mathrm{P}-\mathrm{N}$ bonds of biomolecular substrates. Fluorescent nanoantennas also enabled real-time monitoring of the hydrolysis of $\sim 10-\mathrm{kDa}$ lipopolysaccharides (LPS) (Supplementary Fig. 27) ${ }^{17,31}$. Deriving kinetic parameters for LPS, however, remains challenging due to uncertain sample concentration and the number of phosphates hydrolyzed per LPS molecule ${ }^{68}$.

Fluorescent nanoantennas can also be used to screen for nonproduct inhibitors and activators by monitoring their effect on the AP-mediated hydrolysis of a substrate. For example, we characterized the $K_{\mathrm{i}}$ of five oxyanion inhibitors, as well as the effect of $\mathrm{Mg}^{2+}$, on the hydrolysis of amifostine by AP (Fig. 4 and Supplementary Table 4). The kinetic profiles of phosphate and vanadate agree with the theoretical result for relatively weak and strong competitive inhibitors, respectively (Extended Data Fig. 10).

Characterizing protein conformational states. Other known conformational states of AP can be detected with fluorescent nanoantennas (Fig. 5a). Vanadate $\left(\mathrm{V}_{\mathrm{i}}\right.$; following the same naming format as for $\mathrm{P}_{\mathrm{i}}$ ), for example, is a putative transition state analog (TSA) inhibitor of AP, meaning that it stabilizes a geometry of the enzyme that is reminiscent of the transition state ${ }^{34}$. Nanoantennas detect $\mathrm{V}_{\mathrm{i}}$ binding to bAP via fluorescence quenching (Fig. 5b,c; see control in Supplementary Fig. 30). This quenching response contrasts with the increase in fluorescence observed upon substrate binding to the same site, further indicating that the nanoantennas can efficiently distinguish between highly similar conformational states. Interestingly, tungstate $\left(\mathrm{W}_{\mathrm{i}}\right)$, also recently proposed as a TSA inhibitor of $\mathrm{AP}^{35}$, seems to induce a distinct conformational change as evidenced by the increase in fluorescence (Fig. $5 \mathrm{c}$ ). In contrast, $\mathrm{P}_{\mathrm{i}}$ and molybdate $\left(\mathrm{Mo}_{\mathrm{i}}\right)$, which likewise bind at the active site, did not induce a conformational change detectable by the nanoantennas (Fig. $5 \mathrm{c}$ ). As determined by fluorescence change, the observed dissociation constants $\left(K_{\mathrm{D}}\right)$ of vanadate $(0.54 \pm 0.01 \mu \mathrm{M})$ and tungstate $(7.6 \pm 0.2 \mu \mathrm{M})$ are consistent with their $K_{\mathrm{i}}$ determined using the 'one-shot' fitting of the fluorescent spike (1.2 $\pm 0.3 \mu \mathrm{M}$ and $10.1 \pm 3.5 \mu \mathrm{M}$, respectively; Fig. 4 and Supplementary Table 4), and with the literature ${ }^{69}$.

Nanoantennas can also monitor large conformational changes, such as protein unfolding by thermal denaturation. When attached to SA, nanoantennas display a distinct transition at a melting temperature $\left(T_{\mathrm{M}}\right)$ of $91.7^{\circ} \mathrm{C} \pm 0.7^{\circ} \mathrm{C}$ (Fig. $\left.5 \mathrm{~d}, \mathrm{e}\right)$. This likely represents dissociation of FAM from SA, rather than unfolding of SA or detachment of the whole nanoantenna, since the nanoantenna-SA platform remained stable over this temperature range (Supplementary Fig. 31). With bound bAP, the nanoantennas exhibit a distinct transition at a $T_{\mathrm{M}}$ of $66.4^{\circ} \mathrm{C} \pm 0.1^{\circ} \mathrm{C}$ (Fig. $5 \mathrm{~d}, \mathrm{e}$ ), consistent with the unfolding temperature of AP (Supplementary Figs. 31 and 32$)^{37}$. One potential application of $T_{\mathrm{M}}$ determination by fluorescent nanoantennas could be the characterization of a specific protein in the presence of others (Supplementary Fig. 33). The nanoantennas further enabled derivation of the apparent Gibbs free energy $(\Delta G)$ for the thermal unfolding of bAP $\left(\Delta G=-7.8 \pm 0.5 \mathrm{kcal} \mathrm{mol}^{-1}\right.$ at $T=37^{\circ} \mathrm{C}$; Supplementary Fig. 34).

Rapid screening of nanoantennas. We explored the potential universality of the nanoantenna strategy by using a different model protein that involves protein-protein interaction. As a proof-of-concept, we employed Protein $G$ from streptococcal bacteria, which binds goat immunoglobulin $\mathrm{G}$ (IgG) with high affinity ${ }^{54}$. To facilitate nanoantenna selection, we designed a 96-well-plate screening assay that leverages the convenience of the nanoantenna-SA platform (Fig. 6a,b and Supplementary Note 2). This enabled us to rapidly test 12 nanoantennas with different linker lengths, linker types, chemical connections and fluorophores. We first prepared the plate by adding the different nanoantennas, followed by the addition of SA. We then added biotinylated Protein G (bPG) and recorded the fluorescence intensity in all wells. Upon addition of goat $\operatorname{IgG}$, we observed that nanoantenna 6 (5' T 6-FAM L21 PEG) displayed the largest fluorescence quenching, while nanoantenna 10 ( $3^{\prime}$ Cy3 L12 ssDNA) displayed the largest fluorescence enhancement (Fig. 6c). After identifying these candidate nanoantennas, we confirmed their performance in cuvettes (Fig. 6d,e). This screening strategy also offers an opportunity to further optimize performance via semirational design of the fluorescent nanoantenna. For example, we observed that for this particular protein function, the flexible L21 PEG nanoantenna enabled the best sensitivity for the T 6-FAM fluorophore (Fig. 6c). Although the ssDNA nanoantennas with T 6-FAM were not as sensitive in comparison, we noticed that the longer ones were better than the shorter ones (Fig. 6c). Therefore, we subsequently tested a longer PEG nanoantenna (5' T 6-FAM L41 PEG), not included in our initial screening, and found that it did indeed display improved sensitivity to goat IgG binding (Fig. 6e). Ultimately, however, we selected the Cy3 nanoantenna for subsequent investigations due to its signal-on fluorescence change (Fig. 6e).

In principle, this nanoantenna-SA-bPG complex could be used as a signal-on biosensor to detect the presence of specific types of antibodies (Fig. 6f; bPG $+\operatorname{IgG})^{54}$. Indeed, a sample containing SARS-CoV-2 IgG/IgM led to a similar fluorescence increase (Fig. 6f; bPG + CoV antibody), while it did not respond to a sample negative for SARS-CoV-2 IgG/IgM (Fig. 6f; bPG + Ctrl) nor to the enzyme AP (nonbiotinylated) (Fig. 6f; bPG + AP). Furthermore, swapping bPG for biotinylated Protein A (bPA), which does not bind goat $\mathrm{IgG}^{54}$, also did not display a signal increase (Fig. 6f; bPA + IgG; Supplementary Fig. 35). This control with bPA further shows that the target goat IgG does not nonspecifically interact with the nanoantenna-SA platform. Overall, these results indicate that fluorescent nanoantennas can be rapidly screened for their ability to monitor distinct protein functions.

\section{Discussion}

Here, we have introduced the use of fluorescent nanoantennas as a strategy to monitor protein dynamics. A platform and linker mediate dye-protein interactions via a high local concentration. Protein conformational changes affecting the dye's chemical environment generate a change in the fluorescence signal. By tuning linker length and dye, we have leveraged this strategy to monitor the functions of three proteins: streptavidin, alkaline phosphatase and Protein G. Several observations supported our proposed signaling mechanism. First, FAM nanoantennas detected all conformational changes in their surroundings: their binding to SA, subsequent binding of biotin or a biotinylated protein to SA, and the function of that protein. Most interestingly, the nanoantenna also detected five distinct conformational states of AP: its ground state, enzyme-substrate complexes with various substrates, the distinct conformational changes induced by vanadate ${ }^{34}$ and tungstate ${ }^{35}$ binding, and its unfolded state. Second, 16 structurally distinct substrates of AP, all hydrolyzed by the same mechanism ${ }^{33}$, exhibited similar fluorescence signatures. This fluorescence signature enabled easy characterization of Michaelis-Menten kinetic parameters of all tested substrates and inhibitors. Third, nanoantennas employing chemically diverse dyes that bind to different locations on AP differed in sensitivity but displayed the same kinetics during substrate hydrolysis. Fourth, the strategy was not limited to a single fluorescent dye, since FAM nanoantennas were optimal for monitoring bAP function while the Cy3 nanoantenna was best for bPG. Finally, MD simulations also suggested a signaling mechanism based on conformational change.

A main advantage of fluorescent nanoantennas is their convenience. For example, the nanoantennas can be used with accessible and straightforward fluorescence spectroscopy, as opposed to 
specialized techniques. Furthermore, various conjugation strategies can be developed to facilitate nanoantenna-protein preparation. For instance, here we developed and exploited the modular biotin-SA platform. This requires only nonspecific biotinylation of the protein of interest, as opposed to site-specific attachment chemistry of fluorophores. Indeed, lysine residues can be nonspecifically biotinylated with a simple commercially available kit. While it cannot be assumed to be necessarily true in all cases, biotinylation often does not affect protein function ${ }^{57}$. In comparison, the more complex site-specific labeling needed for FRET strategies has been found to perturb the function of $\beta$-lactamases ${ }^{3}$ and dihydrofolate reductase ${ }^{70}$. In the case where biotinylation would affect a protein's function, other modular attachment strategies could also be developed; for example, one could envisage the use of $\mathrm{N}$ or $\mathrm{C}$ terminus affinity $\operatorname{tags}^{71}$. When employing a modular attachment strategy, efficient nanoantennas can also be rapidly screened using a 96-well plate, as we demonstrated with bPG.

Another important advantage of fluorescent nanoantennas is their versatility. Nanoantennas can be used to monitor distinct biomolecular mechanisms in real time, including small and large conformational changes-in principle, any event that can affect the dye's fluorescence emission. Furthermore, since nanoantennas can distinguish between unbound and substrate-bound enzyme conformations, they can supplant nonnatural colorimetric ${ }^{42}$ and fluorogenic $^{43,44}$ substrates, as well as laborious assays for spectroscopically silent substrates ${ }^{47}$. For example, nanoantennas enable real-time, 'one-shot' kinetic characterization of any substrate, such as ATP and amifostine ${ }^{18-21,29}$. In contrast, standard methods to determine $K_{\mathrm{M}}$ and $k_{\text {cat }}$ for spectroscopically silent substrates require approximately ten measurements at different substrate concentrations ${ }^{47,72}$. They also compare favorably with other 'one-shot' strategies that require microfluidics to generate a range of substrate concentrations, in addition to a fluorescent product ${ }^{73}$. Nanoantennas, however, do have some limitations. For example, unlike other noteworthy techniques $^{7,8,63,64,74-77}$, nanoantennas cannot quantify specific distance variations. Also, not all dye-protein combinations generate a signal change during protein function; some proteins might not work with any dyes. However, looking to the future, we believe that the universality of the nanoantenna strategy may be improved by screening a larger library of dyes and by further exploring the predictive potential of docking and MD simulations. We anticipate that our fluorescent nanoantennas will find exciting applications in the study of protein structure and function and in high-throughput screening.

\section{Online content}

Any methods, additional references, Nature Research reporting summaries, source data, extended data, supplementary information, acknowledgements, peer review information; details of author contributions and competing interests; and statements of data and code availability are available at https://doi.org/10.1038/ s41592-021-01355-5.

Received: 26 February 2021; Accepted: 10 November 2021; Published online: 30 December 2021

\section{References}

1. Hammes-Schiffer, S. \& Klinman, J. Emerging concepts about the role of protein motion in enzyme catalysis. Acc. Chem. Res. 48, 899-899 (2015).

2. Lobb, R. R. \& Auld, D. S. Determination of enzyme mechanisms by radiationless energy transfer kinetics. Proc. Natl Acad. Sci. USA 76, 2684-2688 (1979).

3. Au, H.-W., Tsang, M.-W., So, P.-K., Wong, K.-Y. \& Leung, Y.-C. Thermostable $\beta$-lactamase mutant with its active site conjugated with fluorescein for efficient $\beta$-lactam antibiotic detection. ACS Omega 4, 20493-20502 (2019).

4. Vallée-Bélisle, A. \& Michnick, S. W. Visualizing transient protein-folding intermediates by tryptophan-scanning mutagenesis. Nat. Struct. Mol. Biol. 19, 731-736 (2012).
5. Gregorio, G. G. et al. Single-molecule analysis of ligand efficacy in $\beta_{2}$ AR-G-protein activation. Nature 547, 68-73 (2017).

6. Comstock, M. J. et al. Direct observation of structure-function relationship in a nucleic acid-processing enzyme. Science 348, 352-354 (2015).

7. Hwang, H. \& Myong, S. Protein induced fluorescence enhancement (PIFE) for probing protein-nucleic acid interactions. Chem. Soc. Rev. 43, 1221-1229 (2014).

8. Lerner, E. et al. Toward dynamic structural biology: two decades of singlemolecule Förster resonance energy transfer. Science 359, eaan1133 (2018).

9. Chen, Y., Tsao, K. \& Keillor, J. W. Fluorogenic protein labelling: a review of photophysical quench mechanisms and principles of fluorogen design. Can. J. Chem. 93, 389-398 (2015).

10. Unnikrishnan, B., Wu, R.-S., Wei, S.-C., Huang, C.-C. \& Chang, H.-T. Fluorescent carbon dots for selective labeling of subcellular organelles. ACS Omega 5, 11248-11261 (2020).

11. Eisenmesser, E. Z., Bosco, D. A., Akke, M. \& Kern, D. Enzyme dynamics during catalysis. Science 295, 1520-1523 (2002).

12. Ma, X., Hortelão, A. C., Patiño, T. \& Sánchez, S. Enzyme catalysis to power micro/nanomachines. ACS Nano 10, 9111-9122 (2016).

13. Abou-Zied, O. K. \& Sulaiman, S. A. J. Site-specific recognition of fluorescein by human serum albumin: a steady-state and time-resolved spectroscopic study. Dyes Pigment. 110, 89-96 (2014).

14. Pisoni, D. S. et al. Symmetrical and asymmetrical cyanine dyes. Synthesis, spectral properties, and BSA association study. J. Org. Chem. 79, 5511-5520 (2014).

15. Millán, J.L. Mammalian Alkaline Phosphatases: From Biology to Applications in Medicine and Biotechnology (John Wiley \& Sons, 2006).

16. Lallès, J.-P. Recent advances in intestinal alkaline phosphatase, inflammation, and nutrition. Nutr. Rev. 77, 710-724 (2019).

17. Bates, J. M., Akerlund, J., Mittge, E. \& Guillemin, K. Intestinal alkaline phosphatase detoxifies lipopolysaccharide and prevents inflammation in zebrafish in response to the gut microbiota. Cell Host Microbe 2, 371-382 (2007).

18. Malo, M. S. et al. Intestinal alkaline phosphatase promotes gut bacterial growth by reducing the concentration of luminal nucleotide triphosphates. Am. J. Physiol. Gastrointest. Liver Physiol. 306, G826-G838 (2014).

19. Mizumori, M. et al. Intestinal alkaline phosphatase regulates protective surface microclimate $\mathrm{pH}$ in rat duodenum. J. Physiol. 587, 3651-3663 (2009).

20. Giatromanolaki, A., Sivridis, E., Maltezos, E. \& Koukourakis, M. I. Down-regulation of intestinal-type alkaline phosphatase in the tumor vasculature and stroma provides a strong basis for explaining amifostine selectivity. Semin. Oncol. 29, 14-21 (2002).

21. Hofer, M. et al. Two new faces of amifostine: protector from DNA damage in normal cells and inhibitor of DNA repair in cancer cells. J. Med. Chem. 59, 3003-3017 (2016).

22. Riedel, C. et al. The heat released during catalytic turnover enhances the diffusion of an enzyme. Nature 517, 227-230 (2015).

23. Tsai, L.-C. et al. Expression and regulation of alkaline phosphatases in human breast cancer MCF-7 cells. Eur. J. Biochem. 267, 1330-1339 (2000).

24. Rao, S. R. et al. Tumour-derived alkaline phosphatase regulates tumour growth, epithelial plasticity and disease-free survival in metastatic prostate cancer. Br. J. Cancer 116, 227-236 (2017).

25. Hung, H.-Y. et al. Preoperative alkaline phosphatase elevation was associated with poor survival in colorectal cancer patients. Int. J. Colorectal Dis. 32, 1775-1778 (2017)

26. Namikawa, T. et al. Prognostic significance of serum alkaline phosphatase and lactate dehydrogenase levels in patients with unresectable advanced gastric cancer. Gastric Cancer 22, 684-691 (2019).

27. Kaliannan, K. et al. Intestinal alkaline phosphatase prevents metabolic syndrome in mice. Proc. Natl Acad. Sci. USA 110, 7003-7008 (2013).

28. José, L. M. \& Michael, P. W. Alkaline Phosphatase and Hypophosphatasia. Calcif. Tissue Int. 98, 398-416 (2016)

29. Waymire, K. G. et al. Mice lacking tissue non-specific alkaline phosphatase die from seizures due to defective metabolism of vitamin B-6. Nat. Genet. 11, 45-51 (1995).

30. Park, J.-B. et al. Serum alkaline phosphatase is a predictor of mortality, myocardial infarction, or stent thrombosis after implantation of coronary drug-eluting stent. Eur. Heart J. 34, 920-931 (2012).

31. Yang, W. H. et al. Recurrent infection progressively disables host protection against intestinal inflammation. Science 358, eaao5610 (2017).

32. To, K. K.-W. et al. Temporal profiles of viral load in posterior oropharyngeal saliva samples and serum antibody responses during infection by SARS-CoV-2: an observational cohort study. Lancet Infect. Dis. 20, 565-574 (2020).

33. Stec, B., Holtz, K. M. \& Kantrowitz, E. R. A revised mechanism for the alkaline phosphatase reaction involving three metal ions. J. Mol. Biol. 299, 1303-1311 (2000).

34. Holtz, K. M., Stec, B. \& Kantrowitz, E. R. A model of the transition state in the alkaline phosphatase reaction. J. Biol. Chem. 274, 8351-8354 (1999). 
35. Peck, A., Sunden, F., Andrews, L. D., Pande, V. S. \& Herschlag, D. Tungstate as a transition state analog for catalysis by alkaline phosphatase. J. Mol. Biol. 428, 2758-2768 (2016)

36. Roston, D., Demapan, D. \& Cui, Q. Leaving group ability observably affects transition state structure in a single enzyme active site. J. Am. Chem. Soc. 138, 7386-7394 (2016)

37. Bortolato, M., Besson, F. \& Roux, B. Role of metal ions on the secondary and quaternary structure of alkaline phosphatase from bovine intestinal mucosa. Proteins 37, 310-318 (1999).

38. Ásgeirsson, B., Markússon, S., Hlynsdóttir, S. S., Helland, R. \& Hjörleifsson, J. G. X-ray crystal structure of Vibrio alkaline phosphatase with the non-competitive inhibitor cyclohexylamine. Biochem. Biophys. Rep. 24, $100830(2020)$

39. Aziz, H. et al. Synthesis, characterization, in vitro tissue-nonspecific alkaline phosphatase (TNAP) and intestinal alkaline phosphatase (IAP) inhibition studies and computational evaluation of novel thiazole derivatives. Bioorg. Chem. 102, 104088 (2020)

40. Kiffer-Moreira, T. et al. Catalytic signature of a heat-stable, chimeric human alkaline phosphatase with therapeutic potential. PLoS ONE 9, e89374 (2014).

41. Jiang, Y., Li, X. \& Walt, D. R. Single-molecule analysis determines isozymes of human alkaline phosphatase in serum. Angew. Chem. Int. Ed. Engl. 59, 18010-18015 (2020).

42. Bessey, O. A., Lowry, O. H. \& Brock, M. J. A method for the rapid determination of alkaline phosphatase with five cubic millimeters of serum. J. Biol. Chem. 164, 321-329 (1946).

43. Fernley, H. \& Walker, P. Kinetic behaviour of calf-intestinal alkaline phosphatase with 4-methylumbelliferyl phosphate. Biochem. J. 97, 95-103 (1965).

44. Deng, J., Yu, P., Wang, Y. \& Mao, L. Real-time ratiometric fluorescent assay for alkaline phosphatase activity with stimulus responsive infinite coordination polymer nanoparticles. Anal. Chem. 87, 3080-3086 (2015).

45. Sanzhaeva, U. et al. Imaging of enzyme activity by electron paramagnetic resonance: concept and experiment using a paramagnetic substrate of alkaline phosphatase. Angew. Chem. Int. Ed. Engl. 57, 11701-11705 (2018).

46. Gyurcsányi, R. E., Bereczki, A., Nagy, G., Neuman, M. R. \& Lindner, E. Amperometric microcells for alkaline phosphatase assay. Analyst 127, 235-240 (2002).

47. Baykov, A. A., Evtushenko, O. A. \& Avaeva, S. M. A malachite green procedure for orthophosphate determination and its use in alkaline phosphatase-based enzyme immunoassay. Anal. Biochem. 171, 266-270 (1988).

48. Liu, Y. \& Schanze, K. S. Conjugated polyelectrolyte-based real-time fluorescence assay for alkaline phosphatase with pyrophosphate as substrate. Anal. Chem. 80, 8605-8612 (2008).

49. Liu, Y. et al. Selective sensing of phosphorylated peptides and monitoring kinase and phosphatase activity with a supramolecular tandem assay. J. Am. Chem. Soc. 140, 13869-13877 (2018).

50. Wang, Y., Wang, G., Moitessier, N. \& Mittermaier, A. K. Enzyme kinetics by isothermal titration calorimetry: allostery, inhibition, and dynamics. Front. Mol. Biosci. 7, 583826 (2020).

51. Di Trani, J. M., Moitessier, N. \& Mittermaier, A. K. Complete kinetic characterization of enzyme inhibition in a single isothermal titration calorimetric experiment. Anal. Chem. 90, 8430-8435 (2018).

52. Honarmand Ebrahimi, K., Hagedoorn, P.-L., Jacobs, D. \& Hagen, W. R. Accurate label-free reaction kinetics determination using initial rate heat measurements. Sci. Rep. 5, 16380 (2015).

53. Zhang, L., Buchet, R. \& Azzar, G. Phosphate binding in the active site of alkaline phosphatase and the interactions of 2-nitrosoacetophenone with alkaline phosphatase-induced small structural changes. Biophys. J. 86, 3873-3881 (2004).

54. Akerström, B., Brodin, T., Reis, K. \& Björck, L. Protein G: a powerful tool for binding and detection of monoclonal and polyclonal antibodies. J. Immunol. 135, 2589-2592 (1985).

55. Kada, G., Falk, H. \& Gruber, H. J. Accurate measurement of avidin and streptavidin in crude biofluids with a new, optimized biotin-fluorescein conjugate. Biochim. Biophys. Acta 1427, 33-43 (1999).
56. Buranda, T. et al. Ligand receptor dynamics at streptavidin-coated particle surfaces: a flow cytometric and spectrofluorimetric study. J. Phys. Chem. B 103, 3399-3410 (1999).

57. Iyer, A., Chandra, A. \& Swaminathan, R. Hydrolytic enzymes conjugated to quantum dots mostly retain whole catalytic activity. Biochim. Biophys. Acta Gen. Subj. 1840, 2935-2943 (2014).

58. Fairhead, M., Krndija, D., Lowe, E. D. \& Howarth, M. Plug-and-play pairing via defined divalent streptavidins. J. Mol. Biol. 426, 199-214 (2014).

59. Neish, C. S., Martin, I. L., Henderson, R. M. \& Edwardson, J. M. Direct visualization of ligand-protein interactions using atomic force microscopy. Br. J. Pharmacol. 135, 1943-1950 (2002).

60. Deetanya, P. et al. Interaction of 8-anilinonaphthalene-1-sulfonate with SARS-CoV-2 main protease and its application as a fluorescent probe for inhibitor identification. Comput. Struct. Biotechnol. J. 19, 3364-3371 (2021).

61. Chen, H., Ahsan, S. S., Santiago-Berrios, M. E. B., Abruña, H. D. \& Webb, W. W. Mechanisms of quenching of Alexa fluorophores by natural amino acids. J. Am. Chem. Soc. 132, 7244-7245 (2010).

62. Togashi, D. M., Szczupak, B., Ryder, A. G., Calvet, A. \& O’Loughlin, M. Investigating tryptophan quenching of fluorescein fluorescence under protolytic equilibrium. J. Phys. Chem. A 113, 2757-2767 (2009).

63. Nguyen, B., Ciuba, M. A., Kozlov, A. G., Levitus, M. \& Lohman, T. M. Protein environment and DNA orientation affect protein-induced Cy3 fluorescence enhancement. Biophys. J. 117, 66-73 (2019).

64. Rashid, F. et al. Initial state of DNA-Dye complex sets the stage for protein induced fluorescence modulation. Nat. Commun. 10, 2104 (2019).

65. Marras, S. A. E., Kramer, F. R. \& Tyagi, S. Efficiencies of fluorescence resonance energy transfer and contact-mediated quenching in oligonucleotide probes. Nucleic Acids Res. 30, e122 (2002).

66. Zimmerle, C. T. \& Frieden, C. Analysis of progress curves by simulations generated by numerical integration. Biochem. J. 258, 381-387 (1989).

67. Palmier, M. O. \& Van Doren, S. R. Rapid determination of enzyme kinetics from fluorescence: overcoming the inner filter effect. Anal. Biochem. 371, 43-51 (2007).

68. Komazin, G. et al. Substrate structure-activity relationship reveals a limited lipopolysaccharide chemotype range for intestinal alkaline phosphatase. J. Biol. Chem. 294, 19405-19423 (2019).

69. Ziegler, A. J., Florian, J., Ballicora, M. A. \& Herlinger, A. W. Alkaline phosphatase inhibition by vanadyl- $\beta$-diketone complexes: electron density effects. J. Enzym. Inhib. Med. Chem. 24, 22-28 (2009).

70. Chen, S. et al. Detection of dihydrofolate reductase conformational change by FRET using two fluorescent amino acids. J. Am. Chem. Soc. 135, 12924-12927 (2013)

71. Schwaminger, S. P. et al. Immobilization of PETase enzymes on magnetic iron oxide nanoparticles for the decomposition of microplastic PET. Nanoscale Adv. 3, 4395-4399 (2021).

72. Ritchie, R. J. \& Prvan, T. A simulation study on designing experiments to measure the $K_{\mathrm{m}}$ of Michaelis-Menten kinetics curves. J. Theor. Biol. 178, 239-254 (1996)

73. Mao, H., Yang, T. \& Cremer, P. S. Design and characterization of immobilized enzymes in microfluidic systems. Anal. Chem. 74, 379-385 (2002).

74. Gordon, S. E., Munari, M. \& Zagotta, W. N. Visualizing conformational dynamics of proteins in solution and at the cell membrane. eLife 7, e37248 (2018).

75. Pantazis, A., Westerberg, K., Althoff, T., Abramson, J. \& Olcese, R. Harnessing photoinduced electron transfer to optically determine protein sub-nanoscale atomic distances. Nat. Commun. 9, 4738 (2018).

76. Jarecki, BrianW. et al. Tethered spectroscopic probes estimate dynamic distances with subnanometer resolution in voltage-dependent potassium channels. Biophys. J. 105, 2724-2732 (2013).

77. Mansoor, S. E., DeWitt, M. A. \& Farrens, D. L. Distance mapping in proteins using fluorescence spectroscopy: the tryptophan-induced quenching (TrIQ) method. Biochemistry 49, 9722-9731 (2010).

Publisher's note Springer Nature remains neutral with regard to jurisdictional claims in published maps and institutional affiliations.

(c) The Author(s), under exclusive licence to Springer Nature America, Inc. 2021 


\section{Methods}

Enzymes, substrates and other materials. AP used in this study was from calf intestinal mucosa. Unconjugated AP, bAP, streptavidin-conjugated AP (SA-AP), bPG, bPA and goat IgG (whole molecule) were purchased from Rockland Immunochemicals. SA was from New England Biolabs. VIROTROL SARS-CoV-2 (reactive for SARS-CoV-2 total IgG/IgM and IgG antibodies) and VIROCLEAR SARS-CoV-2 (nonreactive for SARS-CoV-2 total IgG/IgM and IgG antibodies) were from Bio-Rad Laboratories. See Supplementary Information for enzyme storage buffer conditions, as well as details about substrates, inhibitors and other reagents.

Oligonucleotide synthesis. Labeled and unlabeled oligonucleotides were made by standard phosphoramidite chemistry with a solid support DNA/RNA H-6 Synthesizer from K\&A Laborgeräte. Purification of strands with a $5^{\prime}$ protecting group $\left(4,4^{\prime}\right.$-dimethoxytrity (DMT)) was performed with a P-8 oligonucleotide purifier. Strands without a protecting group (for example, 6-FAM and 5-FAM) were purified using high performance liquid chromatography (HPLC) with a 1260 Infinity HPLC instrument from Agilent. The mobile phase was $0.1 \mathrm{M}$ triethylamine with increasing concentration of acetonitrile, and the stationary phase was an XBridge Oligonucleotide BEH C18 OBD Prep Column, $130 \AA$, $2.5 \mu \mathrm{m}$, $10 \mathrm{~mm} \times 50 \mathrm{~mm}$ from Waters Corporation. Extinction coefficients at $260 \mathrm{~nm}$ were predicted using the OligoAnalyzer website (https://www.idtdna.com/calc/analyzer) from Integrated DNA Technologies. DNA was then quantified by UV-visible spectroscopy with a Cary 60 from Agilent or a NanoDrop 2000c Spectrophotometer from Thermo Fisher Scientific. Oligonucleotides were prepared as $200 \mu \mathrm{l}, \sim 800 \mu \mathrm{M}$ stock solutions, and used as $1 \mathrm{ml}, 100 \mu \mathrm{M}$ intermediate solutions; all stored at $-20^{\circ} \mathrm{C}$.

Fluorescence. Fluorescence spectroscopy was recorded with a Cary Eclipse Fluorescence Spectrophotometer from Agilent. For measurements in quartz cuvettes, it was equipped with a Peltier Thermostatted Multicell Holder Accessory from Agilent. For the plate reader measurements, it was equipped with a Microplate Reader ACCY from Varian and used Nunc MaxiSorp 350 $\mu$ l Black 96-well plates from Thermo Fisher Scientific. Fluorescence spectra were recorded with Scan software, fluorescence kinetics with Kinetics software and melting temperatures with Thermal software (Agilent). Typical settings were: excitation (ex)/emission (em) slit widths $5 \mathrm{~nm}$, excitation $498 \mathrm{~nm}$ and emission $520 \mathrm{~nm}$ for FAM (wavelengths denoted hereafter in the format 498/520), CAL 540/561, carboxyrhodamine (ROX) 575/602, carboxytetramethylrhodamine (TAMRA) 565/580, Cyanine 3 (Cy3) 546/563, Quasar 570 (Q570) 550/570, Quasar 670 (Q670) 644/670, Pulsar 650 (P650) 460/650 and methylene blue (MB) 670/690. The photomultiplier tube detector voltage was typically $635 \mathrm{~V}$ for $150 \mathrm{nM}$ fluorescent nanoantennas, but $800 \mathrm{~V}$ for those with MB or P650, as well as $800 \mathrm{~V}$ for experiments with $15 \mathrm{nM}$ FAM nanoantennas. For plate reader measurements, it was $600 \mathrm{~V}$. For kinetics, we typically used averaging time $3.0 \mathrm{~s}$, cycle $0.04 \mathrm{~min}$. For spectra, we typically used CAT mode with 10 scans at 'medium' speed (scan rate $600 \mathrm{~nm} \mathrm{~min}^{-1}$, averaging time $0.1 \mathrm{~s}$, data interval $1 \mathrm{~nm}$ ).

A typical study of nanoantenna fluorescence emission over time (for example, Fig. 1a) was as follows. The intermediate nanoantenna stock solution was added to buffer in a quartz cuvette $(150 \mathrm{nM})$, followed by waiting $5-10 \mathrm{~min}$ for the fluorescence signal to equilibrate. After observing a stable signal, we performed subsequent additions of complementary DNA, proteins, substrates, etc. Final volume at addition of substrate was $1 \mathrm{ml}$. We typically mixed by rapidly pipetting $\sim 10 \times$ using $\sim 50 \mu$ l volume while being careful not to pipette bubbles into the solution. A waiting time of 3-10 min for each step was taken to ensure binding and equilibration. In most cases, cDNA and SA bound very quickly (several seconds), but biotinylated proteins took longer (sometimes up to several minutes). In a typical experiment, we added SA $(50 \mathrm{nM})$ and then bAP $(100 \mathrm{nM})$ for a nanoantenna:SA:bAP ratio of 3:1:2. For some faster lots of enzyme, we added less enzyme, as indicated. Last, we added the substrate (for example, pNPP). To make the effects of dilution negligible, most additions were aliquots of several $\mu \mathrm{l}$.

For dual absorbance and fluorescence kinetics of the same sample, we used a SX20 Stopped Flow Spectrometer from Applied Photophysics with Pro-Data SX software and with a 495-nm cut-off filter. Nanoantenna-protein complex was prepared in one syringe and substrate in another syringe, which were then mixed during the measurement.

Buffer conditions. In our initial studies of the nanoantenna concept with AP and of various dyes (Fig. 1), buffer conditions were $200 \mathrm{mM}$ Tris, $300 \mathrm{mM} \mathrm{NaCl}, 1 \mathrm{mM}$ $\mathrm{MgCl}_{2}, \mathrm{pH} 7.0$ and $37^{\circ} \mathrm{C}$, with $100 \mathrm{nM}$ commercially available bAP and $50 \mathrm{nM} \mathrm{SA}$. A ratio of three nanoantennas per SA, for example, used $150 \mathrm{nM}$ nanoantennas. Later, for comparison with another recent study (Fig. 2) ${ }^{52}$, buffer conditions were $100 \mathrm{mM}$ Tris, $10 \mathrm{mM} \mathrm{NaCl}, \mathrm{pH} 8.0$ and $30^{\circ} \mathrm{C}$, with either $100 \mathrm{nM} \mathrm{bAP}$ (Fig. 2a-c) or $10 \mathrm{nM}$ bAP (Fig. 2d,e; nanoantennas and SA were adjusted proportionally). For characterization of substrates (Fig. 3), the same buffer was used but at $37^{\circ} \mathrm{C}$ with $150 \mathrm{nM}$ nanoantennas, $50 \mathrm{nM} \mathrm{SA}$, commercially available $20 \mathrm{nM}$ bAP and $300 \mu \mathrm{M}$ substrate. Less bAP was used because this lot of bAP enzyme displayed faster substrate hydrolysis kinetics than previous lots that we had purchased. Note that we did observe some enzyme batch-to-batch variation (Supplementary Fig. 26), but the results were still in good agreement with the range of literature values for pNPP. See Supplementary Table 3 for complete kinetic parameters, and Supplementary Figs. 28 and 29 for a discussion of fluorescence baseline. For characterization of effectors

(Fig. 4), the conditions were the same as in Fig. 3, with either $30 \mu \mathrm{M}$ inhibitor or $5 \mathrm{mM}$
$\mathrm{Mg}^{2+}$. For vanadate and related experiments (Fig. 5b,c), the same conditions were also used but with $100 \mathrm{nM}$ of commercially available bAP. In experiments for thermal denaturation of AP (Fig. 5d,e), to reduce $\mathrm{pH}$ variation with temperature, we changed the buffer conditions to $100 \mathrm{mM} \mathrm{NaCl}, 50 \mathrm{mM} \mathrm{Na}_{2} \mathrm{HPO}_{4}, \mathrm{pH} 7.0,37^{\circ} \mathrm{C}$ and used $100 \mathrm{nM}$ of commercially available bAP. For experiments with Protein $\mathrm{G}$ in the 96 -well plate (Fig. 6c), buffer conditions were $200 \mathrm{mM}$ Tris, $300 \mathrm{mM} \mathrm{NaCl}$, pH7.0, room temperature, with $500 \mathrm{nM}$ nanoantennas, $167 \mathrm{nM} \mathrm{SA}, 167 \mathrm{nM} \mathrm{bPG}$ and $\sim 1,000 \mathrm{nM}$ goat IgG. For subsequent experiments with Protein G in cuvettes (Fig. 6e,f), the same buffer was used at $37^{\circ} \mathrm{C}$ with $150 \mathrm{nM}$ nanoantennas, $50 \mathrm{nM} \mathrm{SA}, 50 \mathrm{nM} \mathrm{bPG}$ or bPA and $\sim 500 \mathrm{nM}$ goat IgG, $20 \mu \mathrm{L}$ SARS-CoV-2 antibodies or control (Ctrl) (unknown concentration) or $500 \mathrm{nM}$ AP. Dilution of antibodies was $7.5 \mu \mathrm{l}$ goat IgG in $292.5 \mu \mathrm{l}$ (well plate) or $992.5 \mu \mathrm{l}$ (cuvettes) of buffer, and $20 \mu \mathrm{l}$ VIROTROL SARS-CoV-2 in $980 \mu \mathrm{l}$ (cuvettes) of buffer. Supplementary figures typically used the same conditions as associated experiments in the main text, but see also their captions.

Software. Data analysis was performed in KaleidaGraph from Synergy Software, OriginPro v.9.0 from OriginLab and Microsoft Excel, with all data plotted in KaleidaGraph. The $\log D$ calculations, as a measure of hydrophobicity, were done by MarvinSketch software from ChemAxon. Molecular structure images were also generated with MarvinSketch. Density functional theory (DFT) computations to estimate PEG-based nanoantenna length were done via ChemCompute (https:// chemcompute.org $)^{78}$

Molecular docking simulations. Docking was performed on the SwissDock web server (http://www.swissdock.ch) ${ }^{79,80}$ from the Swiss Institute of Bioinformatics. The 'target' protein structure for streptavidin was PDB 6M9B (Streptomyces avidinii) ${ }^{81}$. Since there was no crystal structure available for the AP used in this study, we instead built a homology model on the SWISS-MODEL web server (https://swissmodel. expasy.org) from the sequence of P19111 (Bos taurus intestinal alkaline phosphatase) and the structure of 1ZEF (Homo sapiens placental alkaline phosphatase) as the template ${ }^{82-84}$. The global model quality estimation was 0.79 , the quaternary structure quality estimate was 0.93 and the identity was 75.52 . 'Ligand' structures (for example, biotin, pNPP, dyes, etc.) were determined to be the major microspecies at $\mathrm{pH} 7.0$ using MarvinSketch software, the manufacturer's product description and available literature (further details in Supplementary Fig. 13), followed by optimization in Avogadro software ${ }^{85}$. Analysis of the docking simulation was done in UCSF Chimera software using the View Dock tool (Type Selection: Dock 4, 5 or 6) ${ }^{86}$. Note that dyes in the simulation did not include the attachment chemistry to the DNA, nor the DNA itself, and are accordingly an estimation of the binding site. Docking simulations were replicated ten times to confirm reproducibility (or lack thereof) for the binding site.

MD simulations. Structure preparation. All protein ligand complexes were prepared using the AP homology model. Two sets of complexes were generated for all three fluorophores (FAM, CAL and Cy3) in complex with or without pNPP substrate. From the docking study of FAM, we chose the best pose ('position') that would have the para and ortho position of the DNA linker attachment point accessible by the solvent. Since AP is a dimer, both binding locations were populated with different ligand conformations for double sampling. In the case of the substrate-bound pNPP/ FAM complex, the FAM ligand was redocked to the AP active site with the substrate present. Again, the best scoring pose of the pNPP/FAM ligand complex from the docking run was chosen. The QuickPrep application of MOE2019 software ${ }^{87}$ with default parameters was used to create a fully parameterized all-atomistic model, which was then used to generate the input files for all MD simulations. Separately, the model of the nanoantenna-SA-bAP complex was built using the streptavidin/ biotin complex (PDB 6M9B) ${ }^{81}$, the AP homology model and the rL12 nanoantenna sequence with $3^{\prime}$-FAM and 5'-T-biotin. The all-atomic model was again generated using the QuickPrep application. A lysine residue in proximity to the AP-binding site was biotinylated and the biotin moiety was placed in the streptavidin active site in a nonclashing conformation. Note that the manufacturer would not disclose the exact composition of the biotin connection to AP (bAP). The DNA linker was constructed using the MOE2019 DNA/RNA builder starting from the crystallized biotin molecule in the neighboring streptavidin active site. Finally, the FAM fluorophore was attached to the $3^{\prime}$-end of the DNA linker and the entire complex energy minimized using the MOE2019 built-in energy minimization application.

MD simulation. The simulation cell and Amber $20^{88}$ input files were generated using MOE2019. The crystallographic water molecules were removed before solvation. Next, the protein/ligand complexes and AP apo structure were embedded in a TIP3P water box with cubic periodic boundary conditions, keeping a distance of $10 \AA$ between the boundaries and the protein. The net charge of the protein was neutralized with $100 \mathrm{mM} \mathrm{NaCl}$. For energy minimization and MD simulations, the Amber14:EHT force field was used and the electrostatic interactions were evaluated by the particle-mesh Ewald method. Each system was energy minimized for 5,000 steps using the Conjugate Gradient method. For equilibration, the system was subjected to a 100 -ps simulation to gradually heat the system from $10 \mathrm{~K}$ to $300 \mathrm{~K}$. Next, a 100-ps NVT ensemble was generated at $300 \mathrm{~K}$, followed by an NPT ensemble for 200 ps at $300 \mathrm{~K}$ and 1 bar. Then, for each complex, a 100 -ns production trajectory was generated for further analysis. The trajectory analysis and frame export for the video was done using scripts shared by the CCG support group. 
Kinetic fitting $\left(\boldsymbol{K}_{\mathrm{v}}, \boldsymbol{k}_{\mathrm{cat}}, \boldsymbol{K}_{\mathrm{i}}\right)$. Fitting was performed using MATLAB (v.R2019a) from MathWorks by following a method with a script obtained from the author ${ }^{51}$ For the script, see 'Script for fitting kinetic data in MATLAB' in the Supplementary Information. Briefly, Michaelis-Menten differential equations with competitive product inhibition (equations (1)-(5)) were integrated using Euler's method with a time step of $\mathrm{d} t=0.1 \mathrm{~s}$, where $K_{\mathrm{M}}$ is the Michaelis-Menten constant, $K_{\mathrm{i}}$ is the product inhibition constant and $k_{\text {cat }}$ is the catalytic rate constant. $[\mathrm{S}]_{t},[\mathrm{P}]_{t}$ and $[\mathrm{ES}]_{t}$ are the concentration of substrate, product and enzyme-substrate complex at time $t$, respectively. Rate dil $_{\text {il }}$ is the rate of dilution of the substrate from the pipette to the cuvette and is estimated to be $2 \mathrm{~s}$ during the dilution and 0 otherwise. $[\mathrm{S}]_{0}$ is the initial concentration of substrate in the syringe before dilution. $[\mathrm{E}]_{0}$ is the concentration of enzyme in the cuvette and is assumed to remain constant throughout the course of the kinetics. Note that substrate addition typically dilutes the enzyme by less than $1 \%$ and is therefore negligible.

$$
\begin{gathered}
\frac{\mathrm{d}[\mathrm{P}]_{t}}{\mathrm{~d} t}=\frac{[\mathrm{E}]_{0} \times k_{\mathrm{cat}} \times[\mathrm{S}]_{t}}{\left(K_{\mathrm{M}}\left(1+\frac{[\mathrm{P}]_{t}}{K_{\mathrm{i}}}\right)+[\mathrm{S}]_{t}\right)} \\
\frac{\mathrm{d}[\mathrm{P}]_{t+\mathrm{d} t}}{\mathrm{~d} t}=[\mathrm{P}]_{t}+\frac{\mathrm{d}[\mathrm{P}]_{t}}{\mathrm{~d} t} \times \mathrm{d} t \\
\frac{\mathrm{d}[\mathrm{S}]_{t}}{\mathrm{~d} t}=-\frac{\mathrm{d}[\mathrm{P}]_{t}}{\mathrm{~d} t}+[\mathrm{S}]_{0} \times \text { Rate }_{\mathrm{dil}} \\
\frac{\mathrm{d}[\mathrm{S}]_{t+\mathrm{d} t}}{\mathrm{~d} t}=[\mathrm{S}]_{t}+\frac{\mathrm{d}[\mathrm{S}]_{t}}{\mathrm{~d} t} \times \mathrm{d} t \\
\frac{\mathrm{d}[\mathrm{ES}]_{t}}{\mathrm{~d} t}=\frac{[\mathrm{E}]_{0} \times[\mathrm{S}]_{t}}{\left(K_{\mathrm{M}}\left(1+\frac{[\mathrm{P}]_{t}}{K_{\mathrm{i}}}\right)+[\mathrm{S}]_{t}\right)}
\end{gathered}
$$

The fluorescence signal was found to be correlated with the concentration of ES and is fit according to equation (6), where the baseline is the native signal of the nanoantenna-SA-bAP complex, $\mathrm{F}_{\max }$ is the fluorescence signal of the nanoantenna-SA-bAP complex when all the enzyme is bound with substrate (that is, high substrate concentration) and $\mathrm{F}_{\text {prod }}$ is the impact of the product concentration on the fluorescence signal of the nanoantenna-SA-bAP complex. Fitting was performed by using the nonlinear least-squares solver lsqcurvefit in MATLAB, which minimizes the sum of the squares of the residuals between the raw data and the computed data. Then, when applicable, the $95 \%$ confidence interval of each parameter is calculated using the nlparci function in MATLAB.

$$
F_{\text {spike }}=\text { Baseline }+F_{\max } \times[\mathrm{ES}]_{t}+F_{\text {prod }} \times[\mathrm{P}]_{t}
$$

Enzymatic equations were sometimes modified to accommodate specific characteristics of some substrates. For example, $\mathrm{PP}_{\mathrm{i}}$ upon cleavage generates two phosphate products, rather than the one phosphate product as generated by pNPP or 4MUP. Therefore, $\mathrm{d}[\mathrm{P}]_{t} / \mathrm{d} t$ was multiplied by 2 . ADP, ATP and GTP can react multiple times and this was considered by multiplying $[\mathrm{S}]_{0}$ by the number of reactive groups. All experiments were done in triplicate.

Preparation of biotinylated AP. For most of this project, we used commercially prepared biotinylated AP. We also prepared our own biotinylated AP to explore lot-to-lot variation issues (used in Fig. 2d and Extended Data Figs. 5 and 6). For this, we used unconjugated AP from Rockland and a Biotin Protein Labeling Kit from Roche Diagnostics. To avoid unwanted side reactions, we removed Tris from the enzyme buffer with a Nanosep Centrifugal Device with Omega Membrane 30K from Pall Corporation by rinsing ten times. Then, we followed the manufacturer's instructions for the biotinylation kit by following 'Procedure 2: Polyclonal antibody' based on the mass of the protein.

Preparation of nanoantenna-AP covalent conjugate. AP was first diluted to $40 \mu \mathrm{M}$ using PBS buffer ( $\mathrm{pH} 7$ ). Then, we added 3 equivalents of freshly prepared SPDP reagent $(20 \mathrm{mM})$ in DMSO. AP was incubated with SPDP solution at room temperature for $30 \mathrm{~min}$. Next, we used a Zeba spin desalting column to exchange the SPDP-modified protein reaction buffer for $10 \mathrm{mM} \mathrm{HEPES}, 150 \mathrm{mM} \mathrm{NaCl}, \mathrm{pH} 8$, and to remove reaction by-products and excess nonreacted SPDP reagent. Separately, we incubated the DNA nanoantenna $\left(5^{\prime} \mathrm{T} 6-\mathrm{FAM}, 3^{\prime} \mathrm{SH}\right)$ with $1 \mathrm{M}$ dithiothreitol (DTT) in $40 \mu \mathrm{TE}$ buffer for $30 \mathrm{~min}$ at $37^{\circ} \mathrm{C}$. Then, we extracted with ethyl acetate and combined the aqueous phases. We then added 8 equivalents of reduced thiol DNA to the SPDP-modified AP and let it react for $1 \mathrm{~h}$ at room temperature. Note that to avoid side reactions, we used a DNA strand that did not contain guanine.

Presentation of data. Error bars on graphs and expressed values represent mean \pm s.e.m. for three distinct measurements. Typically, experiments were performed in triplicate, with the following exceptions: Extended Data Fig. 4 h and Supplementary Fig. 7d for enzyme ratios not near the maximum, as well as Extended Data Fig. 4e,f for covalent attachment of nanoantenna to AP, which had three injections of pNPP to the same sample. All MD simulations were performed once and all molecular docking simulations were performed ten times.

Reporting Summary. Further information on research design is available in the Nature Research Reporting Summary linked to this article.

\section{Data availability}

The fluorescence kinetic signatures of the substrates and inhibitors have been deposited on figshare (https://doi.org/10.6084/m9.figshare.16798174).

\section{Code availability}

The MATLAB fitting script for substrate kinetics is provided in the Supplementary Information.

\section{References}

78. Perri, M. J. \& Weber, S. H. Web-Based Job Submission Interface for the GAMESS Computational Chemistry Program. J. Chem. Educ. 91, 2206-2208 (2014)

79. Grosdidier, A., Zoete, V. \& Michielin, O. SwissDock, a protein-small molecule docking web service based on EADock DSS. Nucleic Acids Res. 39, W270-W277 (2011)

80. Grosdidier, A., Zoete, V. \& Michielin, O. Fast docking using the CHARMM force field with EADock DSS. J. Comput. Chem. 32, 2149-2159 (2011).

81. Basu, S., Finke, A., Vera, L., Wang, M. \& Olieric, V. Making routine native SAD a reality: lessons from beamline X06DA at the Swiss Light Source. Acta Crystallogr. D Struct. Biol. 75, 262-271 (2019).

82. Weissig, H., Schildge, A., Hoylaerts, M. F., Iqbal, M. \& Millán, J. L. Cloning and expression of the bovine intestinal alkaline phosphatase gene: biochemical characterization of the recombinant enzyme. Biochem. J. 290, 503-508 (1993).

83. Llinas, P. et al. Structural studies of human placental alkaline phosphatase in complex with functional ligands. J. Mol. Biol. 350, 441-451 (2005).

84. Harada, T. et al. Characterization of structural and catalytic differences in rat intestinal alkaline phosphatase isozymes. FEBS J. 272, 2477-2486 (2005).

85. Hanwell, M. D. et al. Avogadro: an advanced semantic chemical editor, visualization, and analysis platform. J. Cheminformatics 4, 17 (2012).

86. Pettersen, E. F. et al. UCSF Chimera-a visualization system for exploratory research and analysis. J. Comput. Chem. 25, 1605-1612 (2004).

87. Molecular Operating Environment (MOE) v.2019.01 (Chemical Computing Group, 2019).

88. Case, D. A. et al. Amber 2020 (University of California, San Francisco, 2020).

\section{Acknowledgements}

We acknowledge scholarships from the Natural Sciences and Engineering Research Council of Canada, NSERC (S.G.H., A.D.), Fonds de recherche du Québec-Nature et technologies, FRQNT (S.G.H., D.L.), Groupe de recherche universitaire sur le medicament, GRUM (S.G.H.) and Bourse du Fonds Wilrose Desrosiers et Pauline Dunn (S.G.H.). This work was funded by NSERC, grant numbers RGPIN-2020-06975, RGPIN06403 (A.V.-B.), Canada Reserch Chairs, grant number 950-230012 (A.V.-B.) and Le regroupement québécois de recherche sur la fonction, l'ingénierie et les applications des protéines (PROTEO). We acknowledge past and present members of our laboratory for insightful discussions, particularly C. Prévost-Tremblay (Faculté de médecine, UdeM).

\section{Author contributions}

S.G.H. and A.V.-B. conceived and designed the study. S.G.H. performed all experiments and the molecular docking simulations. A.D. provided ideas in an early phase of the project. D.L. wrote and used the MATLAB script to extract enzyme kinetic parameters. M.C.C.J.C.E. performed molecular dynamics simulations. X.W. realized the synthesis of the nanoantenna-AP conjugate. S.G.H. and A.V.-B. created the figures and wrote the manuscript, which was then reviewed and edited by all of the authors. A.V.-B. provided project oversight and funding.

\section{Competing interests}

The authors declare no competing interests.

\section{Additional information}

Extended data is available for this paper at https://doi.org/10.1038/s41592-021-01355-5.

Supplementary information The online version contains supplementary material available at https://doi.org/10.1038/s41592-021-01355-5.

Correspondence and requests for materials should be addressed to Alexis Vallée-Bélisle.

Peer review information Nature Methods thanks the anonymous reviewers for their contribution to the peer review of this work. Rita Strack was the primary editor on this article and managed its editorial process and peer review in collaboration with the rest of the editorial team.

Reprints and permissions information is available at www.nature.com/reprints. 
a

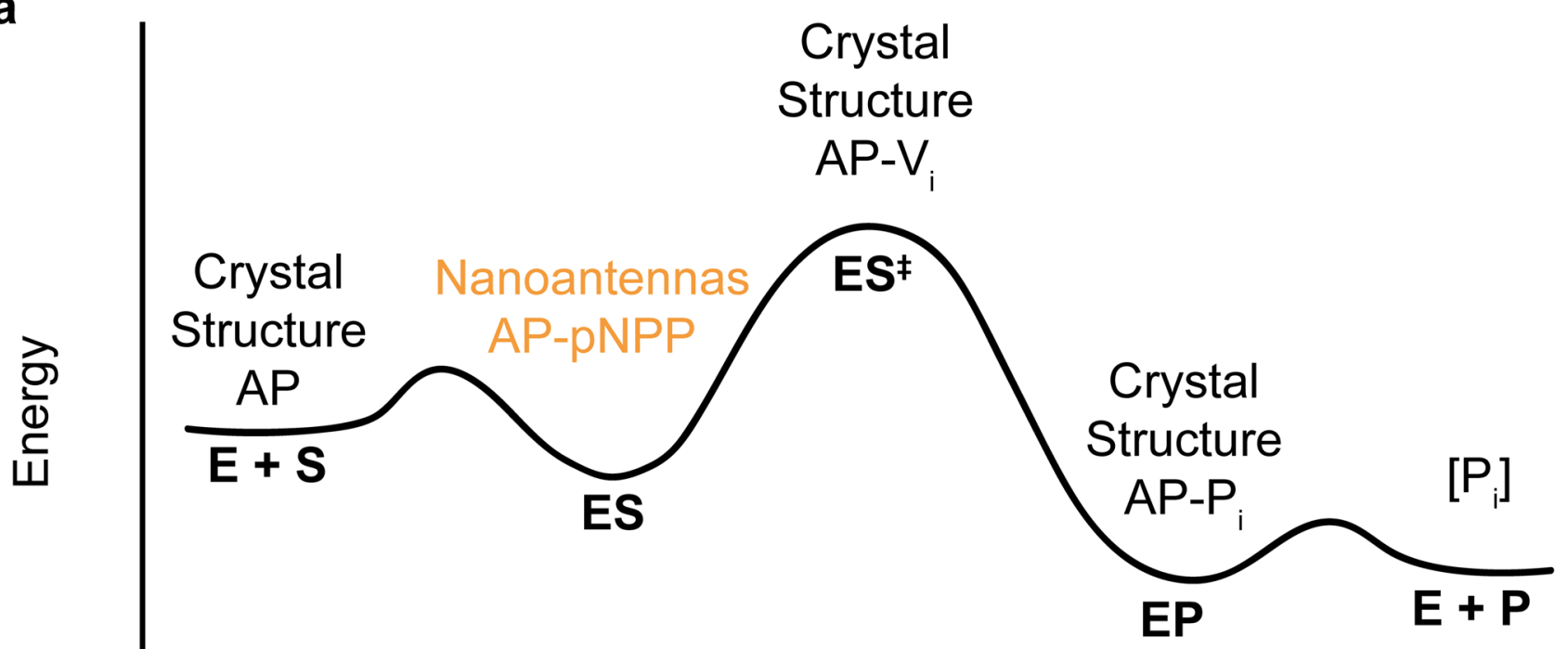

Reaction Coordinate

b

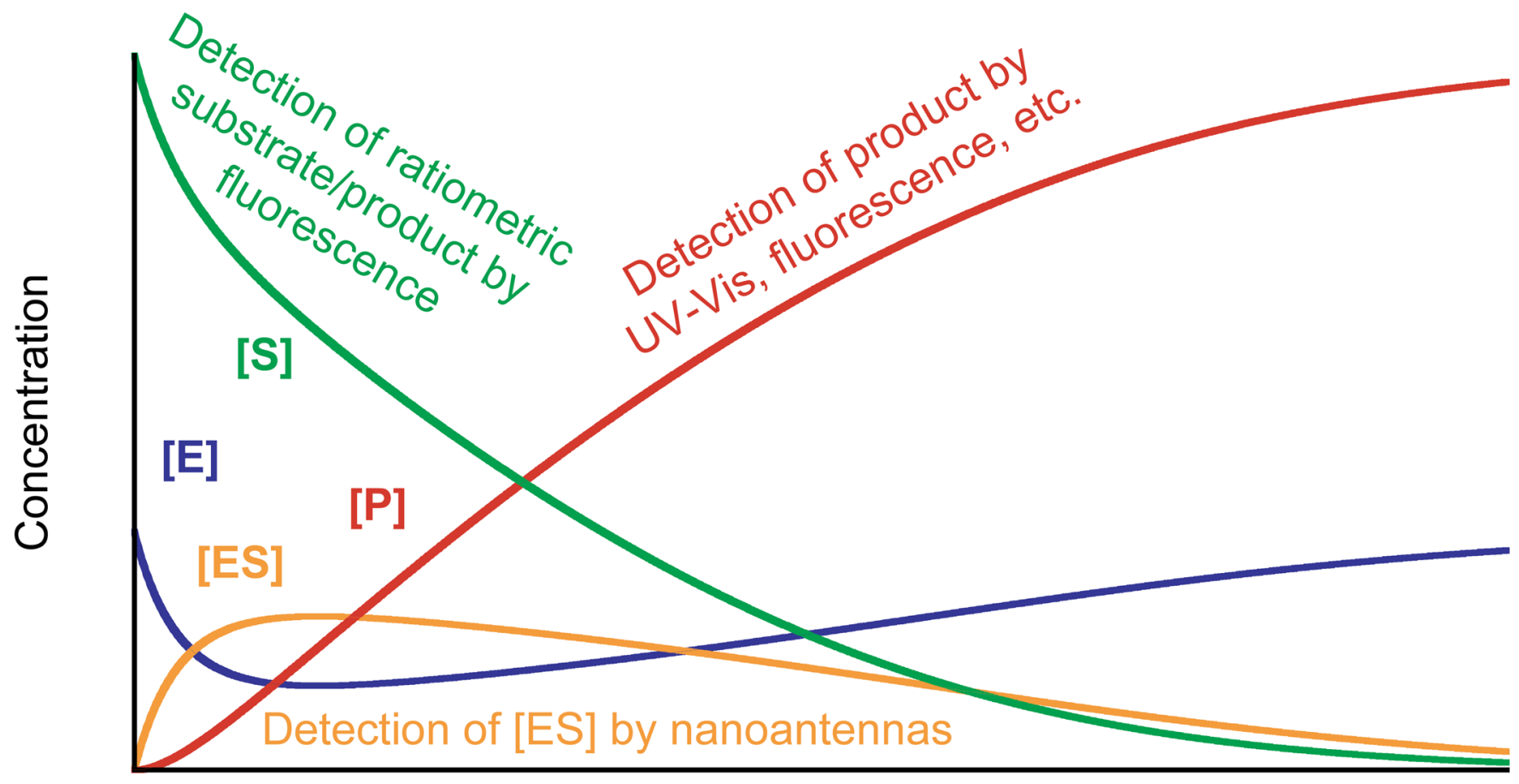

\section{Time}

Extended Data Fig. 1 Progress of a typical enzymatic reaction. (a) The states of an enzyme typically present during the catalysis of a substrate: the enzyme and substrate(s) $(E+S)$ are introduced, followed by formation of the enzyme-substrate complex intermediate (ES). Next is the transition state $(E S)$, followed by the enzyme-product complex intermediate (EP), and finally the enzyme and released product(s) $(E+P)$. (b) Representative changes with time in concentration of the substrate ([S]), product ([P]), enzyme ([E]) and enzyme-substrate complex ([ES]) are shown. See Supplementary Note 1 for further discussion of important studies of methods to detect the different states of AP. 
a

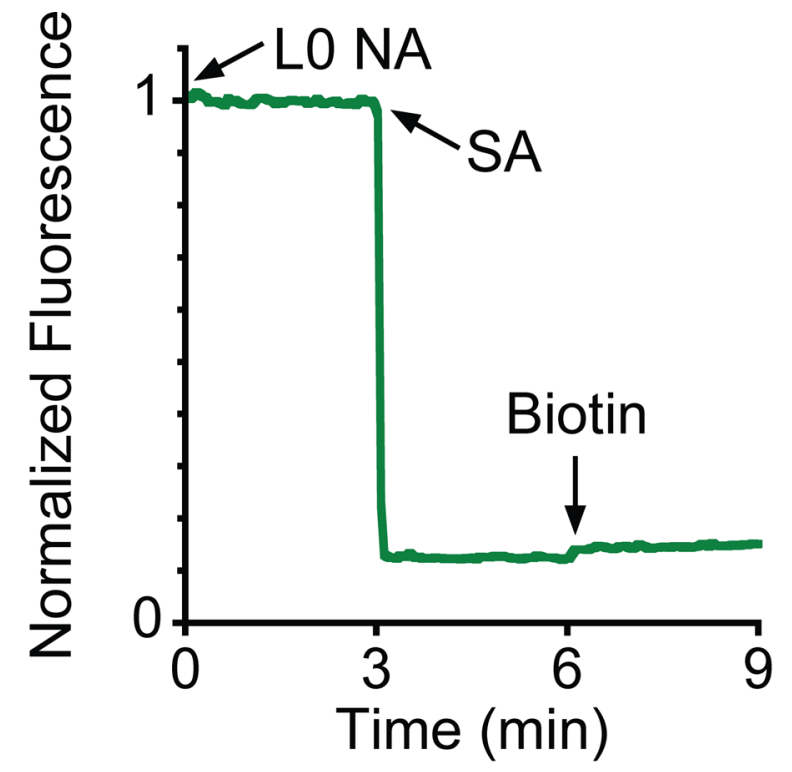

b

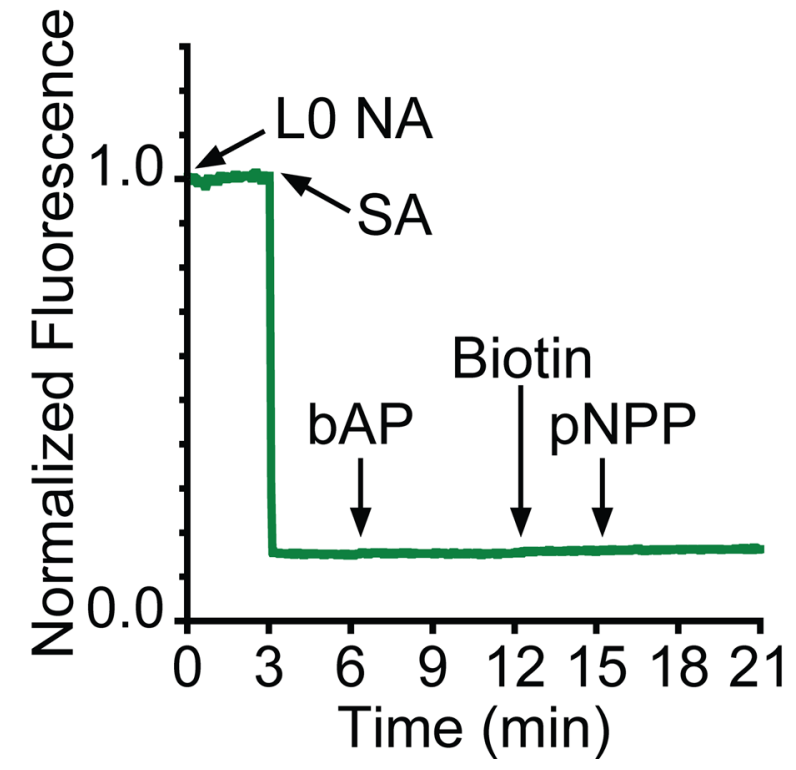

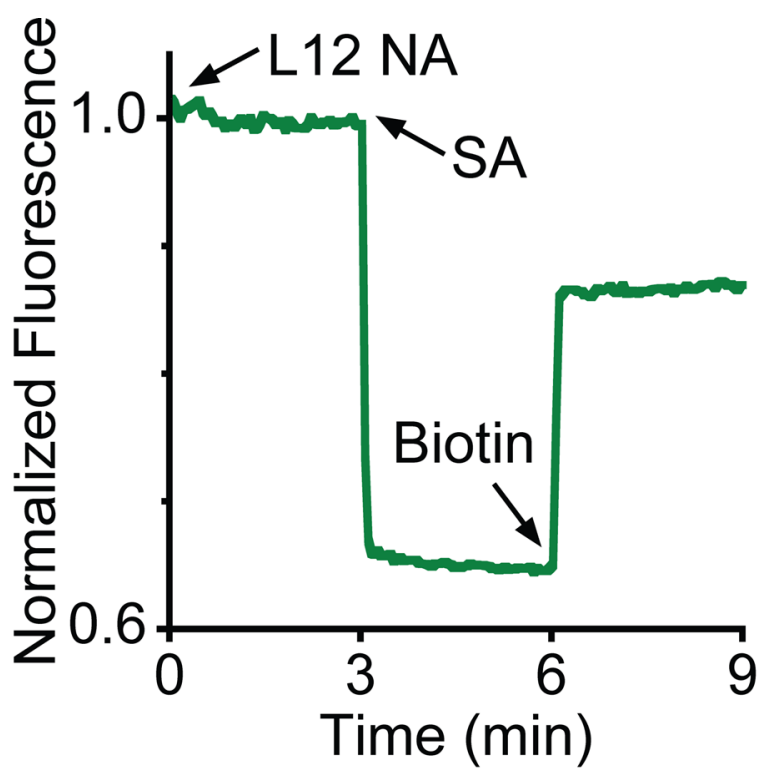

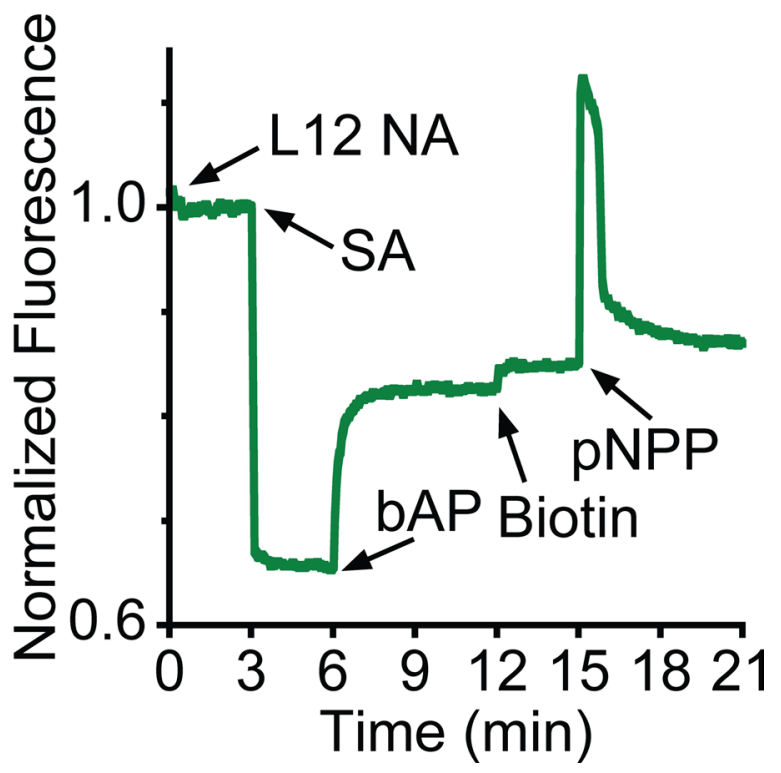

Extended Data Fig. 2 | In contrast to L12, the 'no linker' LO nanoantenna does not allow FAM to bind to unoccupied biotin-binding site on streptavidin nor reach the surface of bAP. (a) Effect of free biotin binding on the nanoantenna-SA platform for the LO (left) and L12 (right) nanoantennas. (b) Effect of free biotin binding on the nanoantenna-SA-bAP complex for the LO and L12 nanoantennas. Discussion: (a) When the LO or L12 nanoantennas bind to SA, their FAM fluorescence is quenched. Next, free biotin is added in excess. The fluorescence of the LO nanoantenna does not change because its FAM moiety cannot reach any unoccupied biotin-binding sites ${ }^{58}$, thus it remains unaffected by biotin binding. In contrast, the fluorescence of the L12 nanoantenna increases because its longer length allows its FAM moiety to weakly bind at an unoccupied biotin-binding site until it is ejected by the incoming higher affinity biotin molecule ${ }^{55,56}$. (b) The LO and L12 nanoantennas bind to SA. bAP then binds to the nanoantenna-SA platform. Next, free biotin is added in excess. The fluorescence of the LO nanoantenna does not change upon addition of bAP because its FAM moiety cannot reach the biotin-binding sites where bAP binds and it also cannot reach the surface of bAP. Subsequent addition of free biotin also does not change its fluorescence for the same reason as without bAP and because most sites are full. In contrast, the fluorescence of the L12 nanoantenna increases upon addition of bAP because its longer length allows its FAM moiety to weakly bind at another biotin-binding site until it is ejected by the incoming high affinity biotin moiety of bAP, thereby allowing it to interact with bAP. Subsequent addition of free biotin does not substantially increase its fluorescence, indicative that the FAM moieties do not remain at the biotin-binding sites of SA. The spike observed upon addition of pNPP confirms that bAP has not been ejected. Although this experiment does not determine whether FAM is now interacting with bAP or with another location on SA, other parts of this paper demonstrate its interaction with bAP (for example, measurement of catalytic function). Conditions: $150 \mathrm{nM}$ nanoantenna, $50 \mathrm{nM}$ SA and $100 \mathrm{nM}$ bAP in $1000 \mathrm{nM}$ biotin in $200 \mathrm{mM}$ Tris, $300 \mathrm{mM} \mathrm{NaCl}, 1 \mathrm{mM} \mathrm{MgCl}$, $\mathrm{pH} 7.0,37^{\circ} \mathrm{C}$. 
$\mathrm{t}=0 \mathrm{~ns}$

\section{streptavidin}

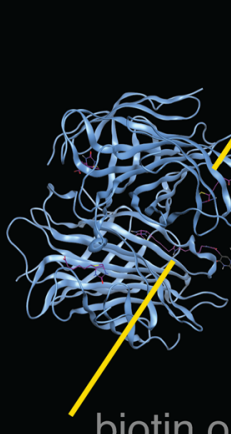

biotin on

nanoantenna

\section{$\mathrm{t}=100 \mathrm{~ns}$}

alkaline phosphatase

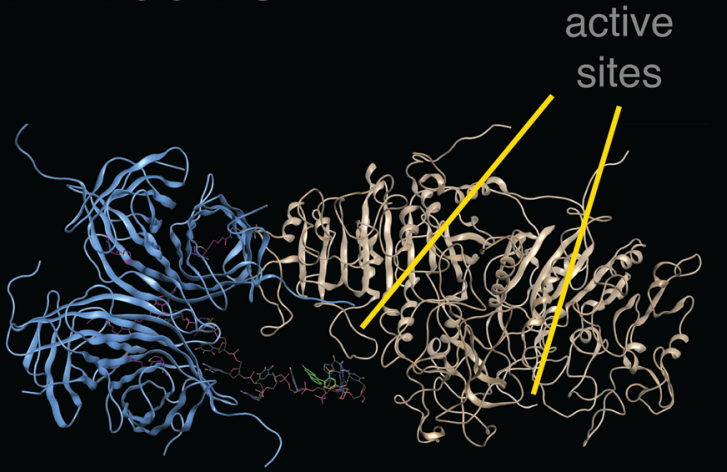

Extended Data Fig. 3 | Molecular dynamics (MD) simulation of a possible nanoantenna-SA-bAP complex. During the simulation, the nanoantenna locates the FAM dye closer to its binding site near the enzyme's substrate active site. See also Video 1. 

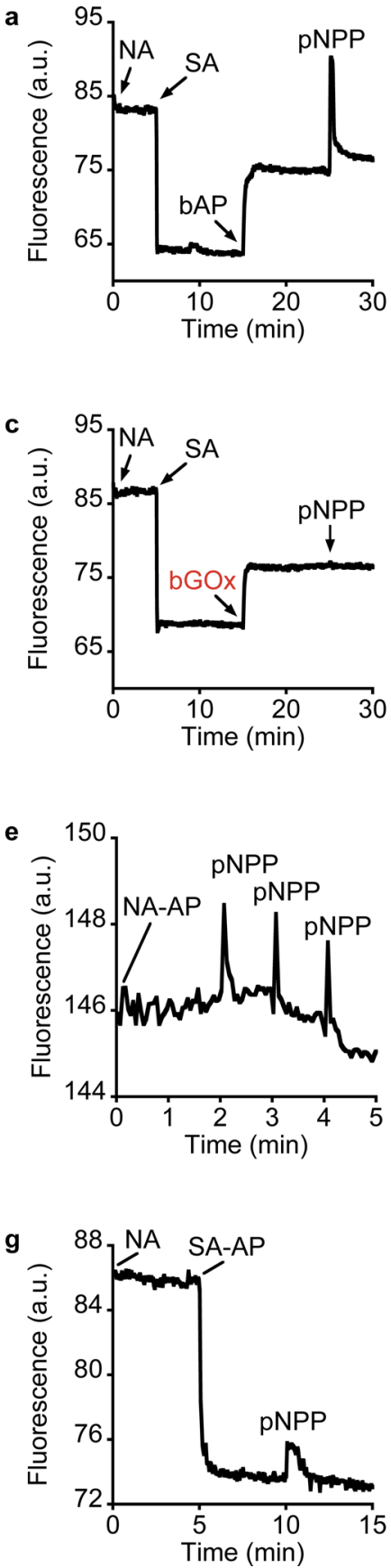
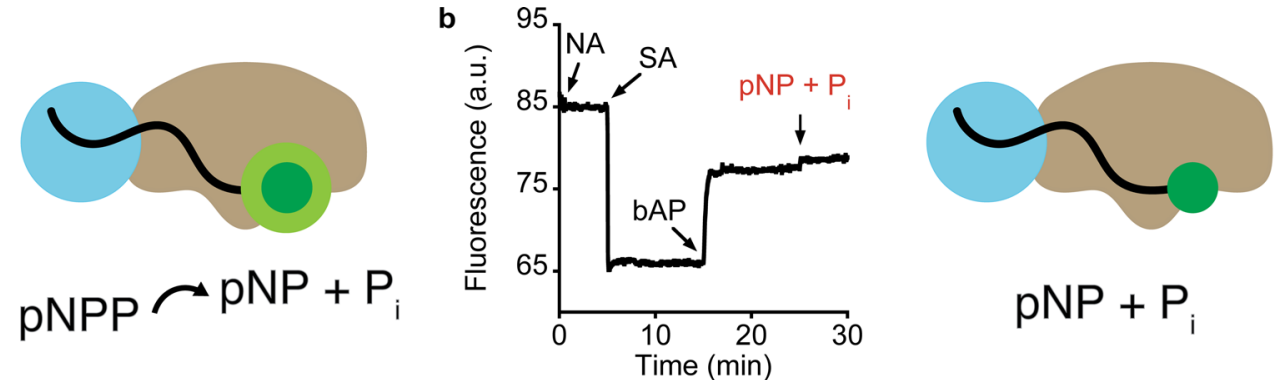
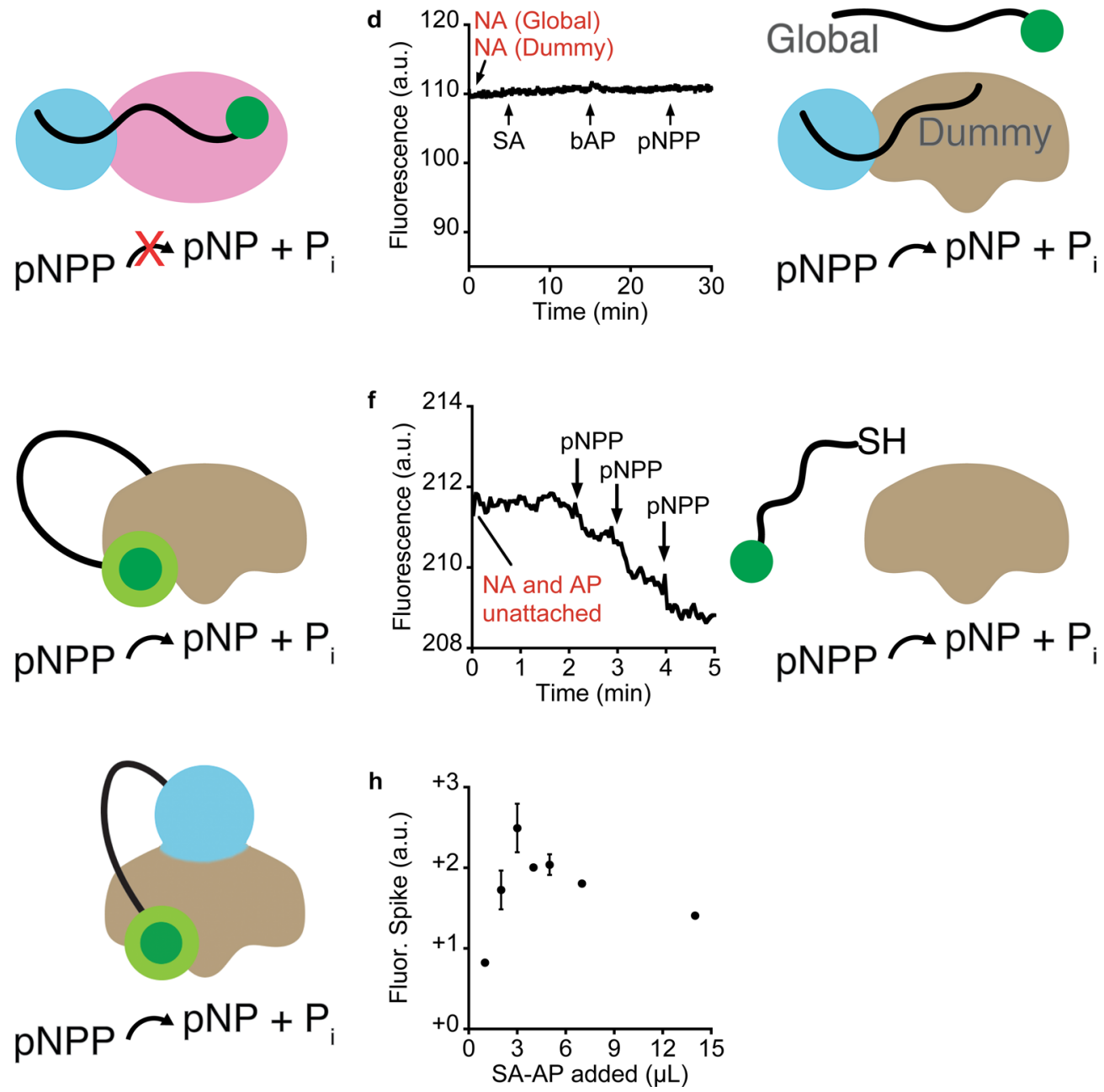

Extended Data Fig. 4 | Fluorescence spikes only occur when there is a hydrolysis reaction and when the nanoantennas are close to the enzyme. (a) Typical nanoantenna fluorescence signal used to monitor pNPP hydrolysis. (b) Addition of the reaction products, $p$-nitrophenol ( $p N P)$ and inorganic phosphate $\left(P_{\mathrm{i}}\right)$, does not give a fluorescence spike because there is no hydrolysis reaction. (c) Using an enzyme without phosphatase activity and which will not hydrolyze pNPP (for example, biotinylated glucose oxidase, bGOx), does not give a fluorescence spike because there is no hydrolysis reaction. (d) Here, the 'Dummy' nanoantenna does not have the dye (that is, no FAM) but it is still attached to SA via its biotin, while the 'Global' nanoantenna has FAM but it is not biotinylated and instead is free in solution. Thus, the hydrolysis reaction of pNPP still occurs, but this system does not monitor it since there is essentially no FAM-bAP interaction. (e) Here, 3'-thiolated L12 ssDNA nanoantennas were covalently attached to the lysine residues of AP (NA-AP; see Online Methods section). This nanoantenna-AP conjugate displays a spike during pNPP hydrolysis similar to the nanoantenna-SA-bAP complex. Although several synthesis steps are involved, it may be desirable for applications for which one does not wish to use the biotin-streptavidin platform. Note that the power was reduced from $635 \mathrm{~V}$ to $450 \mathrm{~V}$ due to the high baseline. (f) As a control, unattached thiolated nanoantennas and unconjugated AP do not display a spike during pNPP hydrolysis. (g) Here, a commercially prepared conjugate of SA covalently attached to AP (SA-AP) was used. The kinetic signature is shown for the PolyT L24 nanoantenna binding to SA-AP that results in fluorescence quenching, followed by pNPP hydrolysis that results in a spike. (h) Without knowledge of the SA-AP molecular weight due to an unknown number of conjugated SAs added by the manufacturer, we instead optimized using SA-AP volume (1, 2, 3, 4, 5, 7 and 14 $\mu \mathrm{L}$ SA-AP). All experiments were performed with $n=1$ biologically independent enzyme samples examined over 3 independent experiments near the apparent maximum ( 2 to $5 \mu \mathrm{L})$ and 1 otherwise. Data are presented as mean values \pm SEM. Even after this simple optimization, however, the spike intensity during pNPP hydrolysis remains weaker compared to using the SA and bAP strategy. Overall, these results show that no matter which attachment strategy is used, and despite some being better than others, FAM will still find its binding site on the AP enzyme. Conditions: (a-d) $150 \mathrm{nM}$ L12 PolyT nanoantenna, $50 \mathrm{nM}$ SA, $150 \mathrm{nM}$ bAP, (e,f) $40 \mathrm{nM}$ nanoantenna-bAP conjugate and (g,h) $150 \mathrm{nM}$ nanoantenna, 1 to $14 \mu \mathrm{LSA}-\mathrm{AP} ; 100 \mu \mathrm{M} \mathrm{pNPP}$ in $200 \mathrm{mM}$ Tris, $300 \mathrm{mM} \mathrm{NaCl}, 1 \mathrm{mM} \mathrm{MgCl}$, $\mathrm{pH}^{7.0,} 37^{\circ} \mathrm{C}$. 

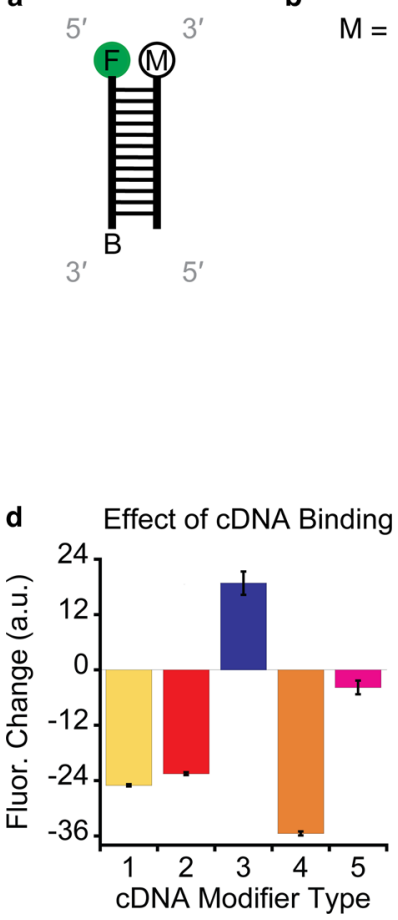

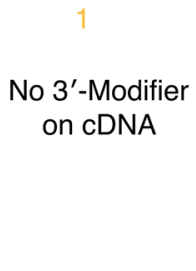

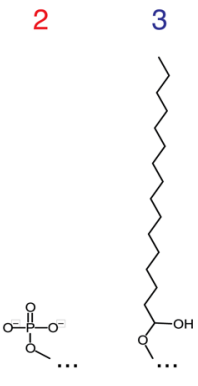

ל
5

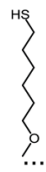

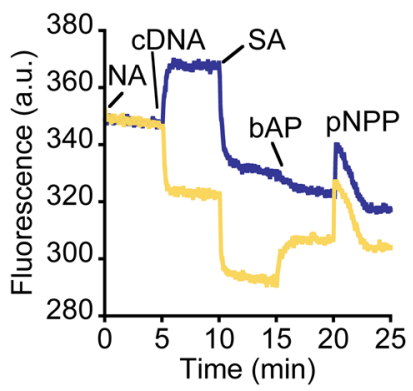

Extended Data Fig. 5 | Effect of various chemical modifiers near FAM on cDNA binding, SA binding, bAP binding, and pNPP hydrolysis. Here, we investigated whether various chemical modifications near the dye ('modifiers') could affect the fluorescence signal of FAM by changing its interaction with bAP. (a) We used the L12 ssDNA FAM nanoantenna (5' T 6-FAM) with a complementary strand containing the modifier located at the 3'-end. (b) For comparison, 1 ) is the cDNA without a modifier; 2 ) is the cDNA with phosphate; 3 ) is the cDNA with a hydrophobic $C_{16}$ alkane chain; 4 ) is the cDNA with a modifier that contains a disulfide that would normally be cleaved before use to provide thiol functionality; 5 ) is a cDNA with the cleaved thiol. (c) Example kinetic signatures and (d) summary of all results. In short, the SA and bAP binding steps display different intensities with each modifier, but nevertheless they are qualitatively similar in all cases (that is, signal up or signal down). The exception to this is the $\mathrm{C}_{16}$ alkane chain, which results in fluorescence quenching when bAP binds. In all cases, the spike intensity during pNPP hydrolysis was similar. All experiments were performed with $n=1$ biologically independent enzyme samples examined over 3 independent experiments. Data are presented as mean values \pm SEM. Conditions: $15 \mathrm{nM}$ nanoantenna, $75 \mathrm{nM}$ cDNA, $5 \mathrm{nM} \mathrm{SA}, 10 \mathrm{nM}$ homemade bAP, $25 \mu \mathrm{M}$ pNPP, pH 8.0, $100 \mathrm{mM}$ Tris, $10 \mathrm{mM} \mathrm{NaCl}, 37^{\circ} \mathrm{C}$. 
a

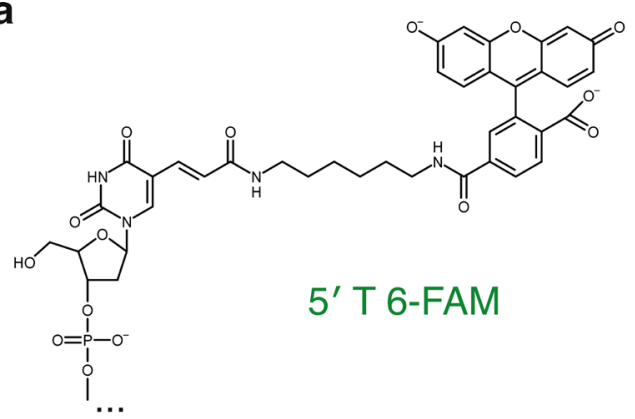<smiles></smiles>

5' 6-FAM

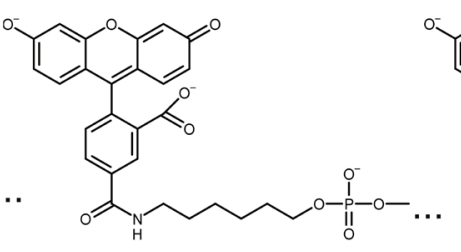<smiles></smiles>

3' 5-FAM

\section{b}

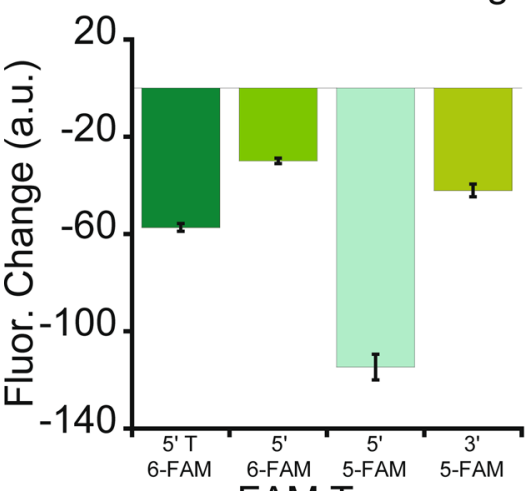

FAM Type

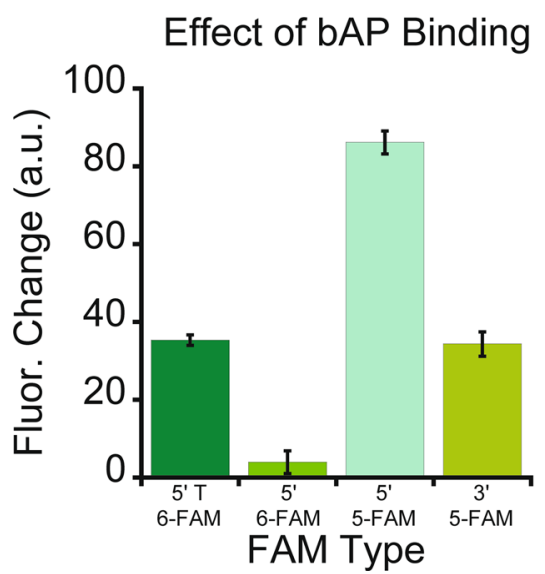

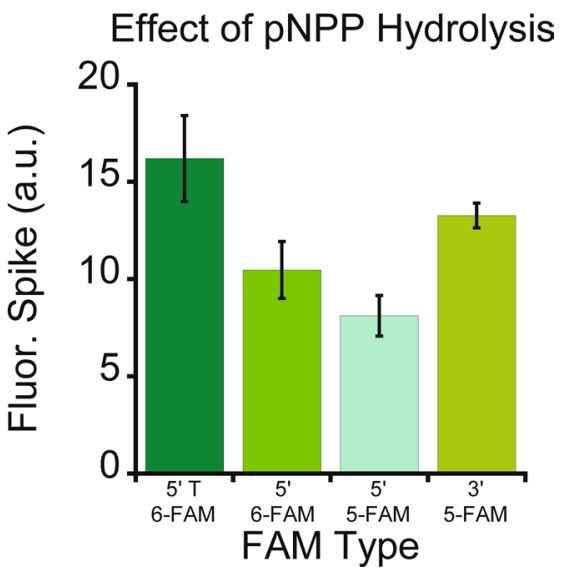

Extended Data Fig. 6 | Effect of FAM connection and isomer on SA binding, bAP binding, and pNPP hydrolysis. (a) In most of this study until this point, we used a L12 ssDNA nanoantenna with 5' thymine 6-carboxyfluorescein (5' T 6-FAM). Here, however, we also tested other FAM connections on the same DNA sequence: 5' 6-carboxyfluorescein (5' 6-FAM), 5' 5-carboxyfluorescein (5' 5-FAM) and 3' 5-carboxyfluorescein (3' 5-FAM). (b) Shown are the quenching of fluorescence upon SA binding, the increase of fluorescence upon bAP binding, and the transient fluorescence spike during pNPP hydrolysis. Despite the similar fluorescence emission of 5-FAM and 6-FAM when conjugated to DNA, the various FAM nanoantennas display different trends for protein binding and pNPP hydrolysis. These differences are likely due to how the chemical connection subtly affects FAM-bAP interaction. All experiments were performed with $n=1$ biologically independent enzyme samples examined over 3 independent experiments. Data are presented as mean values \pm SEM. Conditions: $15 \mathrm{nM}$ nanoantenna, $5 \mathrm{nM} \mathrm{SA}, 10 \mathrm{nM}$ homemade bAP, $30 \mu \mathrm{M} \mathrm{pNPP}, \mathrm{pH} 8.0,100 \mathrm{mM}$ Tris, $10 \mathrm{mM} \mathrm{NaCl}, 30^{\circ} \mathrm{C}$. PMT voltage $=800 \mathrm{~V}$. 


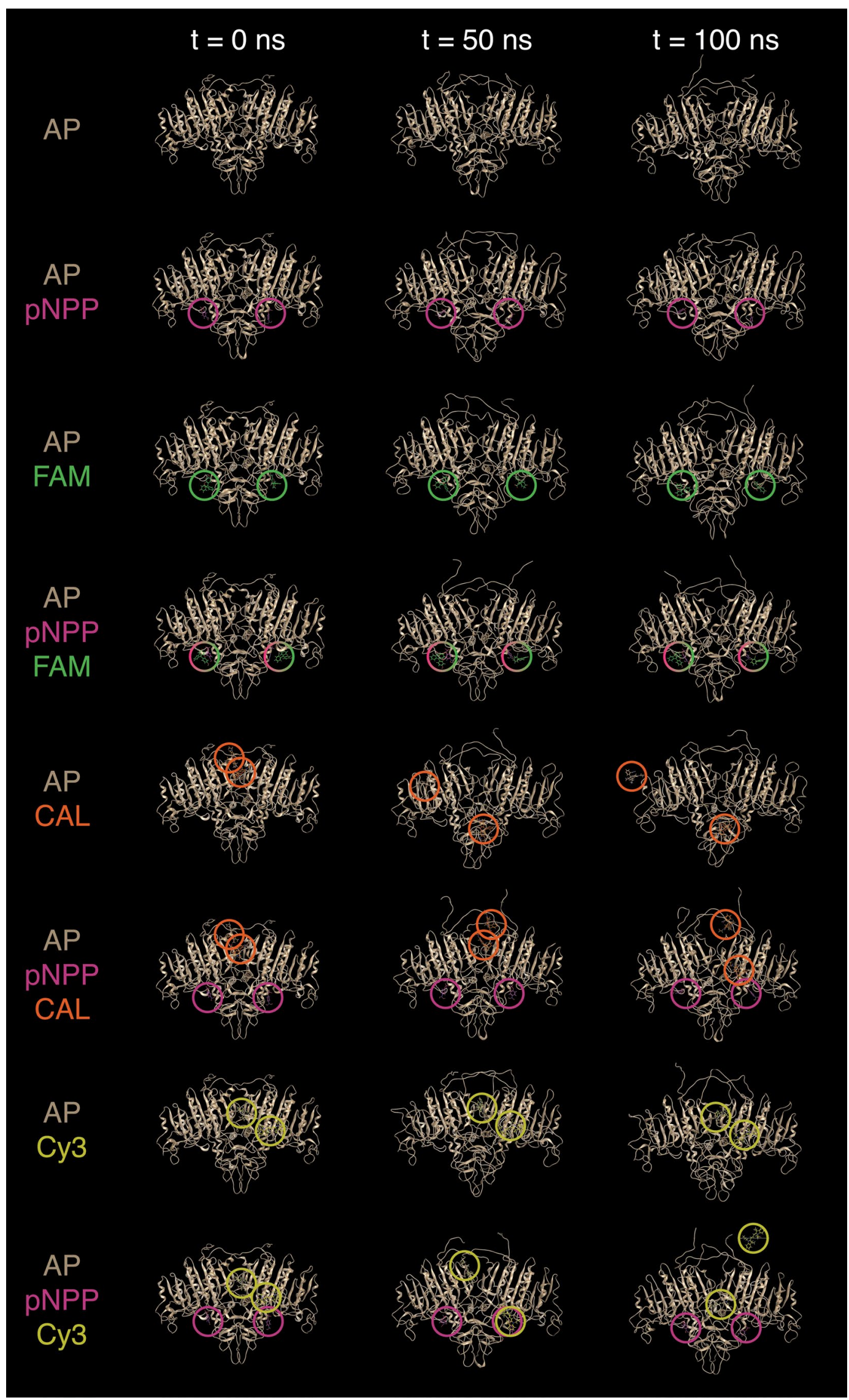

Extended Data Fig. 7 | See next page for caption. 
Extended Data Fig. 7 | Molecular dynamics (MD) trajectories of dyes and/or substrate on AP. The 100 ns MD simulation of AP with or without a dye (FAM, CAL, Cy3) and with or without the substrate pNPP. We selected the lowest energy pose (see Supplementary Fig. 13) and the next lowest energy pose that does not overlap with it and that has the linker location exposed. Dyes and pNPP are circled for visual clarity. In all cases, we observed that pNPP remains bound at the active sites. For AP with FAM and with or without pNPP, the position of FAM remains unchanged. For AP with CAL and with or without pNPP, the CAL dye does not have a stable position in either case. For AP-Cy3 without pNPP, the Cy3 dye position does not change, but with pNPP bound, the dye position can change, and it even dissociates from the surface. We emphasize that these MD simulations represent a possible signaling mechanism (see main text), and not a definite proposal. 
a

DNA-FAM CDNA
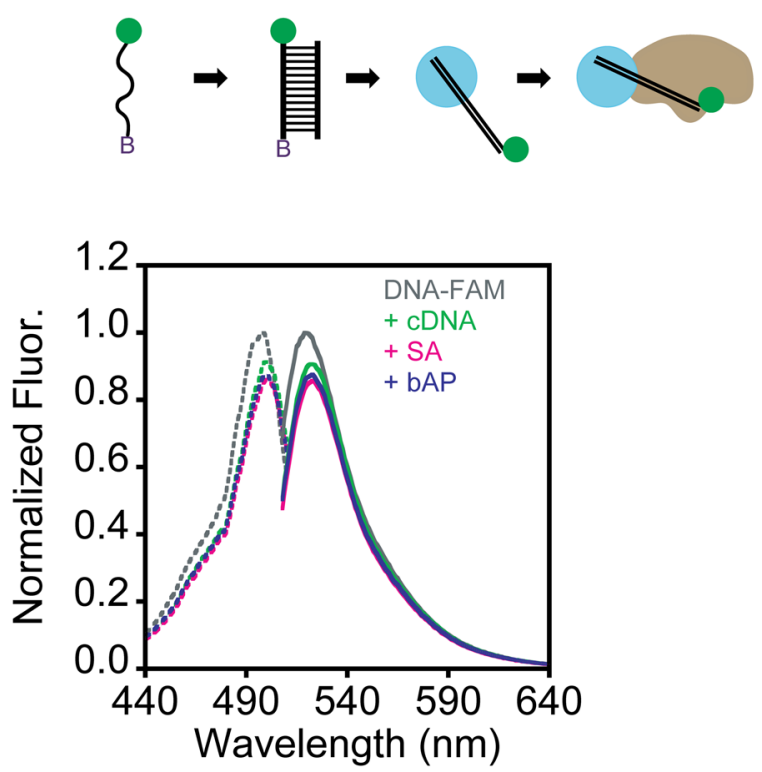

c DNA-FAM CDNA-CAL SA bAP
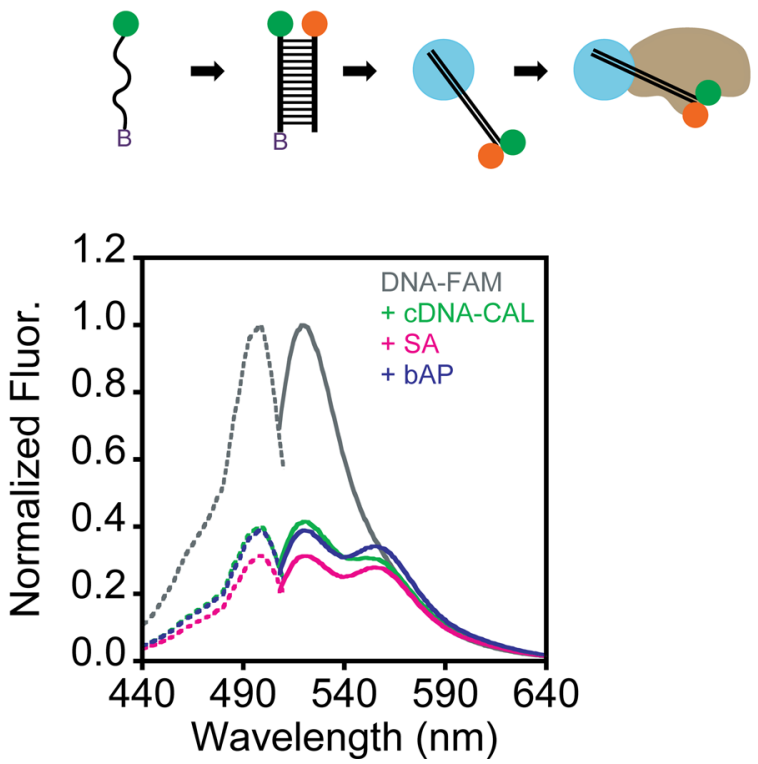

b DNA-CAL CDNA SA bAP
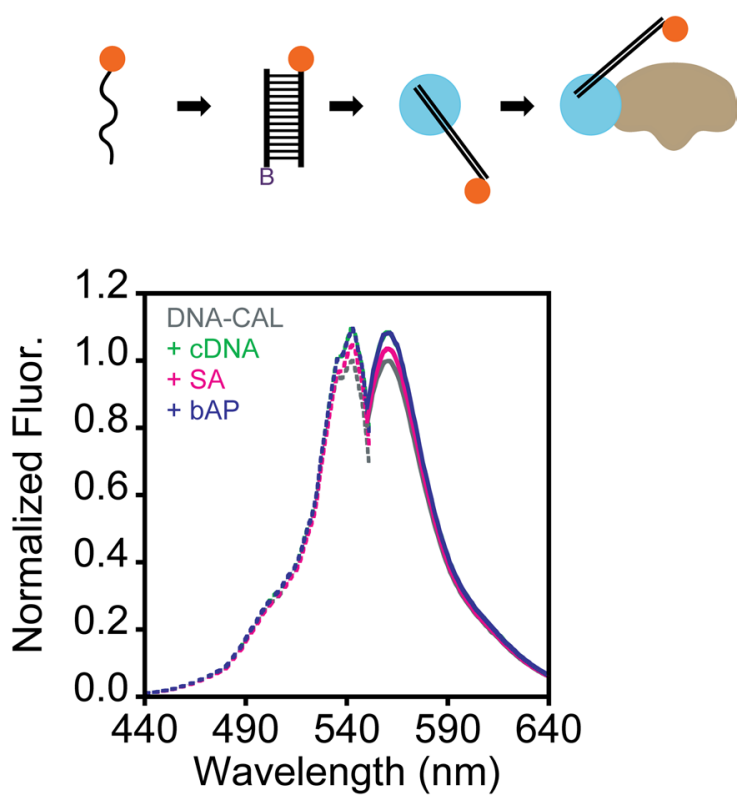

d DNA-CAL CDNA-FAM SA bAP
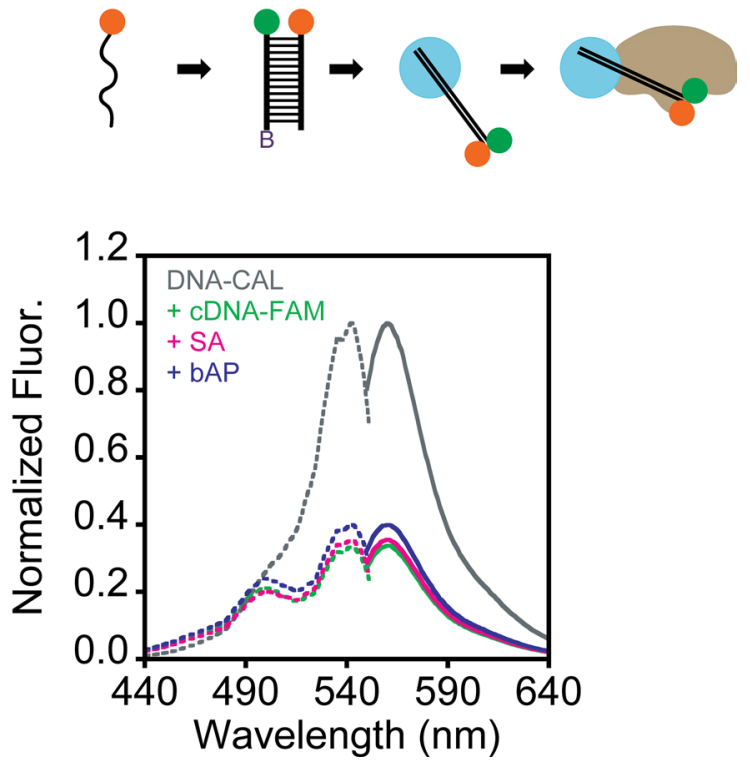

Extended Data Fig. 8 | Excitation and emission spectra of double-dye dsDNA nanoantenna (FAM-CAL) suggest dye stacking. The excitation spectra (dashed line) and emission spectra (solid line) of the formation of the double-dye dsDNA nanoantenna: starting with ssDNA, after binding of cDNA, after binding of SA, and after binding of bAP. In (a), only the FAM dye is present, and excitation and emission of FAM wavelengths $\left(\lambda_{\text {em }}=520 \mathrm{~nm}\right.$ and $\lambda_{\mathrm{ex}}=498 \mathrm{~nm}$ ) are relatively unaffected by the addition of the complementary DNA (of note, the dsDNA antenna displays little sensitivity to SA and bAP attachment relative to ssDNA nanoantennas, see Fig. 1). In (b), only the CAL dye is present, and excitation and emission of CAL wavelengths $\left(\lambda_{\text {em }}=\right.$ $561 \mathrm{~nm}$ and $\lambda_{\mathrm{ex}}=540 \mathrm{~nm}$ ) are relatively unaffected by the addition of the complementary DNA. In (c) and (d), both dyes were present after the cDNA step (that is, the systems were chemically identical), but (c) was measured with the FAM wavelengths and (d) with the CAL wavelengths. We observed that both the FAM and CAL excitation and emission spectra are drastically affected when proximal to the other dye. This remains true even following the addition of the SA and bAP proteins. This decrease of signal intensity is likely attributable to a contact-mediated quenching mechanism between the dyes ${ }^{65}$. These dyes seem to remain stacked even after the nanoantenna has moved to SA and bAP. 

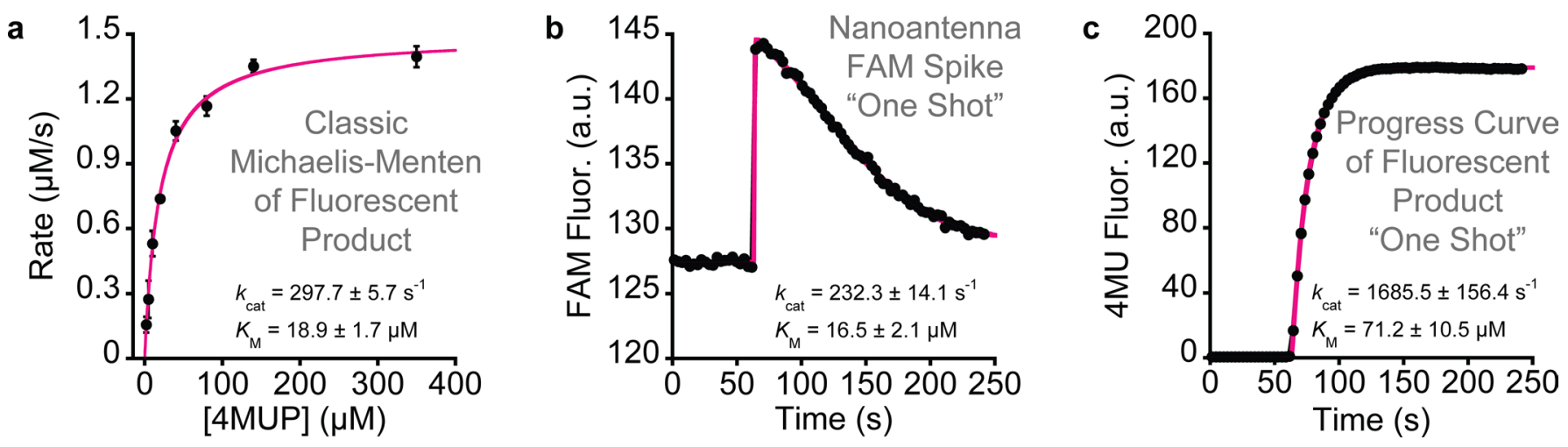

Extended Data Fig. 9 | Comparison of methods and error. (a) Classic Michaelis-Menten method to determined $k_{\text {cat }}$ and $K_{M}$ values for the 4 MUP substrate using the initial rates of $4 \mathrm{MU}$ product generation. (b) Nanoantenna 'one-shot' method to determined $k_{\text {cat }}$ and $K_{M}$ values using the FAM fluorescence spike obtained during 4MUP hydrolysis. The values determined using both methods displayed good agreement. This analysis was performed several months after our values obtained for 4MUP in Fig. 3, which also shows the reproducibility of the method. (c) We further compared the accuracy of the 'one-shot' nanoantenna method over a similar approach performed using the $4 \mathrm{MU}$ progress curve under the same conditions. We found that a 'one shot' $4 \mathrm{MU}$ progress curve over-estimated both the $k_{\text {cat }}$ and $K_{M}$ of the enzyme-substrate system. See relevant literature for fitting of progress curves. Conditions were $100 \mathrm{mM}$ Tris, $10 \mathrm{mM} \mathrm{NaCl}, \mathrm{pH} 8.0,37^{\circ} \mathrm{C}$; also $150 \mathrm{nM}$ nanoantenna, $50 \mathrm{nM} \mathrm{SA}, 20 \mathrm{nM}$ bAP, and $300 \mu \mathrm{M} 4 \mathrm{MUP}$ in (b and $\mathbf{c}$ ), and the same buffer and temperature but $37.5 \mathrm{nM}$ nanoantenna, $12.5 \mathrm{nM}$ SA, $5 \mathrm{nM}$ bAP, and $2 \mu \mathrm{M}, 5 \mu \mathrm{M}, 10 \mu \mathrm{M}, 20 \mu \mathrm{M}, 40 \mu \mathrm{M}, 80 \mu \mathrm{M}, 140 \mu \mathrm{M}$ or $350 \mu \mathrm{M} 4 \mathrm{MUP}$ in (a). In the latter two, the concentration of bAP was reduced to facilitate measurement of low 4MUP concentrations, and the nanoantenna and SA concentrations were reduced proportionately. All experiments were performed with $n=1$ biologically independent enzyme samples examined over 3 independent experiments. (24 measurements for the classic Michaelis-Menten via initial rates and 3 each for the nanoantenna spike and product progress curve). Data are presented as mean values \pm SEM. 

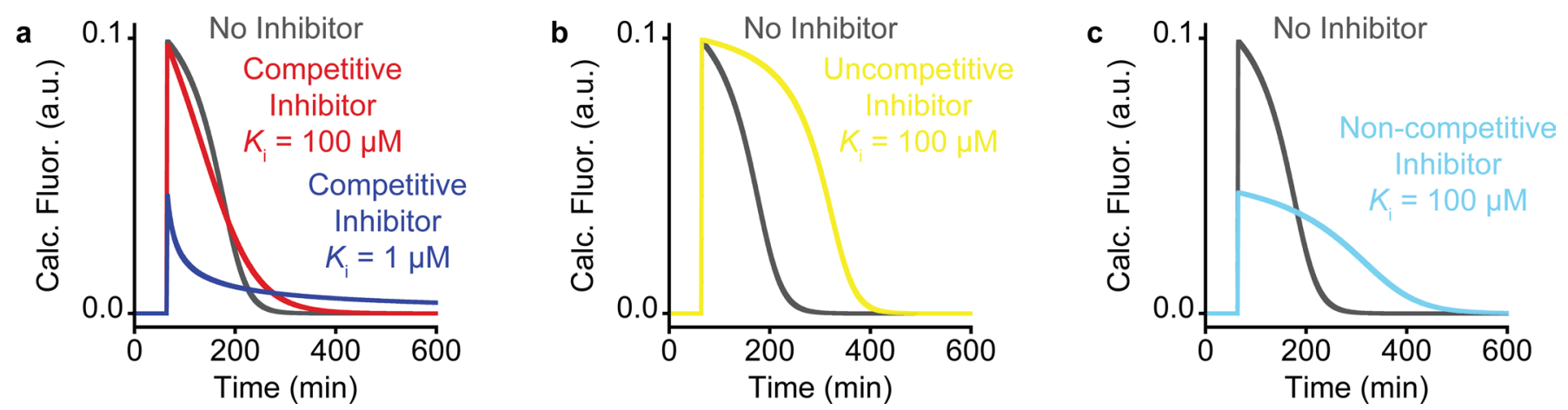

Extended Data Fig. 10 | Theoretical nanoantenna kinetic signatures for different types of inhibitors. Here, we generated the expected spike profile of a theoretical system with the parameters: $k_{\text {cat }}=100 \mathrm{~s}^{-1}, K_{\mathrm{M}}=10 \mu \mathrm{M}$, [enzyme] $=100 \mathrm{nM}$, [substrate] $=1000 \mu \mathrm{M}$, and [inhibitor] $=125 \mu \mathrm{M}$. Shown are the effects of (a) competitive inhibitors with $K_{\mathrm{i}}=100 \mu \mathrm{M}$ and $K_{\mathrm{i}}=1 \mu \mathrm{M}$, (b) uncompetitive inhibitor with $K_{\mathrm{i}}=100 \mu \mathrm{M}$, and (c) non-competitive inhibitor with $K_{\mathrm{i}}=100 \mu \mathrm{M}$. 


\section{Reporting Summary}

Nature Research wishes to improve the reproducibility of the work that we publish. This form provides structure for consistency and transparency in reporting. For further information on Nature Research policies, see our Editorial Policies and the Editorial Policy Checklist.

\section{Statistics}

For all statistical analyses, confirm that the following items are present in the figure legend, table legend, main text, or Methods section.

$\mathrm{n} / \mathrm{a}$ Confirmed

$\bigotimes$ The exact sample size $(n)$ for each experimental group/condition, given as a discrete number and unit of measurement

$\square$ \ A statement on whether measurements were taken from distinct samples or whether the same sample was measured repeatedly

Х The statistical test(s) used AND whether they are one- or two-sided

$\triangle \square$ Only common tests should be described solely by name; describe more complex techniques in the Methods section.

Х $\square$ A description of all covariates tested

Х $\square$ A description of any assumptions or corrections, such as tests of normality and adjustment for multiple comparisons

$\square$ A full description of the statistical parameters including central tendency (e.g. means) or other basic estimates (e.g. regression coefficient) AND variation (e.g. standard deviation) or associated estimates of uncertainty (e.g. confidence intervals)

For null hypothesis testing, the test statistic (e.g. $F, t, r$ ) with confidence intervals, effect sizes, degrees of freedom and $P$ value noted

Х Give $P$ values as exact values whenever suitable.

Х $\square$ For Bayesian analysis, information on the choice of priors and Markov chain Monte Carlo settings

$\bigotimes \square$ For hierarchical and complex designs, identification of the appropriate level for tests and full reporting of outcomes

$\triangle \square$ Estimates of effect sizes (e.g. Cohen's d, Pearson's $r$ ), indicating how they were calculated

Our web collection on statistics for biologists contains articles on many of the points above.

\section{Software and code}

Policy information about availability of computer code

Data collection Cary Eclipse: Scan (v1.2(147)), Kinetics (v1.2(146)), Thermal (v1.2(146)); SX20 Stopped Flow: Pro-Data SX (v2.5.1852.0)

Data analysis Data analysis and plotting: Excel (v2101), KaleidaGraph (v4.0), OriginPro (v9.0.0); Kinetic fitting: MATLAB (vR2019a); Docking, MD or DFT: Amber2020, Avogadro (v1.2.0), MOE2019 (v2019.0102), UCSF Chimera (v1.11; ViewDock tool, file type: Dock 4, 5 or 6); Molecular structure images and $\log D$ calculations: MarvinSketch (v18.9.0)

For manuscripts utilizing custom algorithms or software that are central to the research but not yet described in published literature, software must be made available to editors and

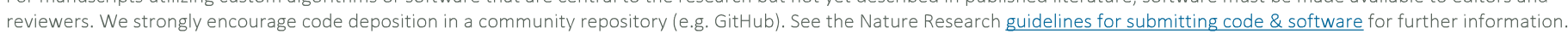

Data

Policy information about availability of data

All manuscripts must include a data availability statement. This statement should provide the following information, where applicable:

- Accession codes, unique identifiers, or web links for publicly available datasets

- A list of figures that have associated raw data

- A description of any restrictions on data availability

The fluorescent signature of all substrates (Fig. 3) and the effect of inhibitors on the fluorescent signature of the substrate amifostine (Fig. 4) are provided on figshare (DOI: 10.6084/m9.figshare.16798174). 
Please select the one below that is the best fit for your research. If you are not sure, read the appropriate sections before making your selection.

\Life sciences

Behavioural \& social sciences

Ecological, evolutionary \& environmental sciences

For a reference copy of the document with all sections, see nature.com/documents/nr-reporting-summary-flat.pdf

\section{Life sciences study design}

All studies must disclose on these points even when the disclosure is negative.
Sample size
We employed triplicate measurements as this is typically the standard employed when performing enzymatic studies.
Data exclusions No data were excluded.
$\begin{array}{ll}\text { Replication } & \begin{array}{l}\text { Fluorescent nanoantenna experiments on the hydrolysis of pNPP were performed dozens of times on different days and under different } \\ \text { conditions. For other substrates, inhibitors and antibodies, all experiments were replicated at least three times. }\end{array}\end{array}$
Randomization This is not relevant to our study. All our attempts to replicate experiments led to similar results.
Blinding Fitting of the data was performed by a different person than the one who collected the data, and was without detailed knowledge of the properties of the substrate or inhibitor employed.

\section{Behavioural \& social sciences study design}

All studies must disclose on these points even when the disclosure is negative.

Study description

Research sample

Sampling strategy

Data collection

Timing

Data exclusions

Non-participation

Randomization

\begin{abstract}
Briefly describe the study type including whether data are quantitative, qualitative, or mixed-methods (e.g. qualitative cross-sectional, quantitative experimental, mixed-methods case study).
\end{abstract}

State the research sample (e.g. Harvard university undergraduates, villagers in rural India) and provide relevant demographic information (e.g. age, sex) and indicate whether the sample is representative. Provide a rationale for the study sample chosen. For studies involving existing datasets, please describe the dataset and source.

Describe the sampling procedure (e.g. random, snowball, stratified, convenience). Describe the statistical methods that were used to predetermine sample size OR if no sample-size calculation was performed, describe how sample sizes were chosen and provide a rationale for why these sample sizes are sufficient. For qualitative data, please indicate whether data saturation was considered, and what criteria were used to decide that no further sampling was needed.

Provide details about the data collection procedure, including the instruments or devices used to record the data (e.g. pen and paper, computer, eye tracker, video or audio equipment) whether anyone was present besides the participant(s) and the researcher, and whether the researcher was blind to experimental condition and/or the study hypothesis during data collection.

Indicate the start and stop dates of data collection. If there is a gap between collection periods, state the dates for each sample cohort.

If no data were excluded from the analyses, state so OR if data were excluded, provide the exact number of exclusions and the rationale behind them, indicating whether exclusion criteria were pre-established.

State how many participants dropped out/declined participation and the reason(s) given $O R$ provide response rate $O R$ state that no participants dropped out/declined participation.

\section{Ecological, evolutionary \& environmental sciences study design}

All studies must disclose on these points even when the disclosure is negative.

Study description

Research sample
Briefly describe the study. For quantitative data include treatment factors and interactions, design structure (e.g. factorial, nested, hierarchical), nature and number of experimental units and replicates.

Describe the research sample (e.g. a group of tagged Passer domesticus, all Stenocereus thurberi within Organ Pipe Cactus National Monument), and provide a rationale for the sample choice. When relevant, describe the organism taxa, source, sex, age range and 
Sampling strategy

any manipulations. State what population the sample is meant to represent when applicable. For studies involving existing datasets, describe the data and its source.

Note the sampling procedure. Describe the statistical methods that were used to predetermine sample size OR if no sample-size calculation was performed, describe how sample sizes were chosen and provide a rationale for why these sample sizes are sufficient.

Data collection

Describe the data collection procedure, including who recorded the data and how.

Timing and spatial scale

Indicate the start and stop dates of data collection, noting the frequency and periodicity of sampling and providing a rationale for these choices. If there is a gap between collection periods, state the dates for each sample cohort. Specify the spatial scale from which the data are taken

Data exclusions

If no data were excluded from the analyses, state so OR if data were excluded, describe the exclusions and the rationale behind them, indicating whether exclusion criteria were pre-established.

Reproducibility

Describe the measures taken to verify the reproducibility of experimental findings. For each experiment, note whether any attempts to repeat the experiment failed $O R$ state that all attempts to repeat the experiment were successful.

Randomization

Describe how samples/organisms/participants were allocated into groups. If allocation was not random, describe how covariates were controlled. If this is not relevant to your study, explain why.

Blinding

Describe the extent of blinding used during data acquisition and analysis. If blinding was not possible, describe why OR explain why blinding was not relevant to your study.

Did the study involve field work? $\square$ Yes $\square$ No

\section{Field work, collection and transport}

Field conditions

Location

Access \& import/export

Disturbance
Describe the study conditions for field work, providing relevant parameters (e.g. temperature, rainfall).

State the location of the sampling or experiment, providing relevant parameters (e.g. latitude and longitude, elevation, water depth).

\section{Reporting for specific materials, systems and methods}

We require information from authors about some types of materials, experimental systems and methods used in many studies. Here, indicate whether each material, system or method listed is relevant to your study. If you are not sure if a list item applies to your research, read the appropriate section before selecting a response.

\begin{tabular}{l|l} 
Materials \& experimental syste \\
\hline$n / a$ & Involved in the study \\
\hline & $\bigotimes$ Antibodies \\
$\square$ & $\square$ Eukaryotic cell lines \\
$\square$ & $\square$ Animals and other organisms \\
$\square$ & $\square$ Human research participants \\
$\square$ & $\square$ Clinical data \\
$\square$ & $\square$ Dual use research of concern
\end{tabular}

\begin{tabular}{l|l}
\multicolumn{2}{l}{ Methods } \\
\hline n/a & Involved in the study \\
$\square$ & $\square$ ChIP-seq \\
$\square$ & $\square$ Flow cytometry \\
$\square$ & $\square$ MRI-based neuroimaging
\end{tabular}

Antibodies

Antibodies used

Goat IgG, Rockland Immunochemicals, lot \# 32090, catalogue \# 005-0102-0010.

VIROTROL SARS-CoV-2, Bio-Rad, Reactive for SARS-CoV-2 total IgG/lgM and IgG antibodies, human plasma based, lot \# 390300 , catalogue \# 200300A

VIROCLEAR SARS-CoV-2, Bio-Rad, Non-reactive for SARS-CoV-2 total IgG/IgM and IgG antibodies, human plasma based, lot \# 390600, catalogue \# 200500.

Validation

Goat IgG, Rockland Immunochemicals: "Goat IgG whole molecule was prepared from normal serum by a multi-step process which includes delipidation, salt fractionation and ion exchange chromatography followed by extensive dialysis against the buffer stated above. Goat IgG whole molecule assayed by immunoelectrophoresis resulted in a single precipitin arc against anti-Goat IgG and antiGoat Serum." Reference provided on website: Yamada, Y. et al. Efficient and high-speed transduction of an antibody into living cells using a multifunctional nanocarrier system to control intracellular trafficking. J. Pharm. Sci. 104, 2845-2854 (2015). https://rocklandinc.com/Product. aspx?id=45482 
VIROTROL SARS-CoV-2 and VIROCLEAR SARS-CoV-2, Bio-Rad:

"These products are unassayed quality controls designed to produce results within a target range established by each laboratory. These products should be analyzed in the same manner as unknown specimens according to instructions supplied by the manufacturer of the test kit being used. Levels of reactivity and specific performance characteristics of these products will vary with different manufacturers' kits and assay procedures."

https://www.bio-rad.com/webroot/web/pdf/cdg/literature/Q-1676-CE.pdf

Eukaryotic cell lines

Policy information about cell lines

Cell line source(s)

State the source of each cell line used.

Authentication

Describe the authentication procedures for each cell line used OR declare that none of the cell lines used were authenticated.

Mycoplasma contamination

Commonly misidentified lines

(See ICLAC register)

Confirm that all cell lines tested negative for mycoplasma contamination OR describe the results of the testing for

mycoplasma contamination $O R$ declare that the cell lines were not tested for mycoplasma contamination.

Name any commonly misidentified cell lines used in the study and provide a rationale for their use.

\section{Palaeontology and Archaeology}

\begin{tabular}{|c|c|}
\hline Specimen provenance & $\begin{array}{l}\text { Provide provenance information for specimens and describe permits that were obtained for the work (including the name of the } \\
\text { issuing authority, the date of issue, and any identifying information). }\end{array}$ \\
\hline Specimen deposition & Indicate where the specimens have been deposited to permit free access by other researchers. \\
\hline Dating methods & $\begin{array}{l}\text { If new dates are provided, describe how they were obtained (e.g. collection, storage, sample pretreatment and measurement), where } \\
\text { they were obtained (i.e. lab name), the calibration program and the protocol for quality assurance OR state that no new dates are } \\
\text { provided. }\end{array}$ \\
\hline
\end{tabular}

Tick this box to confirm that the raw and calibrated dates are available in the paper or in Supplementary Information.

Ethics oversight

Identify the organization(s) that approved or provided guidance on the study protocol, OR state that no ethical approval or guidance was required and explain why not.

Note that full information on the approval of the study protocol must also be provided in the manuscript.

\section{Animals and other organisms}

Policy information about studies involving animals; ARRIVE guidelines recommended for reporting animal research
Laboratory animals
For laboratory animals, report species, strain, sex and age OR state that the study did not involve laboratory animals.
Wild animals
Provide details on animals observed in or captured in the field; report species, sex and age where possible. Describe how animals were caught and transported and what happened to captive animals after the study (if killed, explain why and describe method; if released, say where and when) OR state that the study did not involve wild animals.
Field-collected samples For laboratory work with field-collected samples, describe all relevant parameters such as housing, maintenance, temperature, photoperiod and end-of-experiment protocol OR state that the study did not involve samples collected from the field.
Ethics oversight

Identify the organization(s) that approved or provided guidance on the study protocol, OR state that no ethical approval or guidance
was required and explain why not.

Note that full information on the approval of the study protocol must also be provided in the manuscript.

\section{Human research participants}

Policy information about studies involving human research participants

Population characteristics

Recruitment

Ethics oversight

Note that full information on the approval of the study protocol must also be provided in the manuscript. 
Policy information about clinical studies

All manuscripts should comply with the ICMJE guidelines for publication of clinical research and a completed CONSORT checklist must be included with all submissions.

\begin{tabular}{|c|c|}
\hline Clinical trial registration & Provide the trial registration number from ClinicalTrials.gov or an equivalent agency. \\
\hline Study protocol & Note where the full trial protocol can be accessed OR if not available, explain why. \\
\hline Data collection & Describe the settings and locales of data collection, noting the time periods of recruitment and data collection. \\
\hline Outcomes & Describe how you pre-defined primary and secondary outcome measures and how you assessed these measures. \\
\hline
\end{tabular}

\section{Dual use research of concern}

Policy information about dual use research of concern

Hazards

Could the accidental, deliberate or reckless misuse of agents or technologies generated in the work, or the application of information presented in the manuscript, pose a threat to:

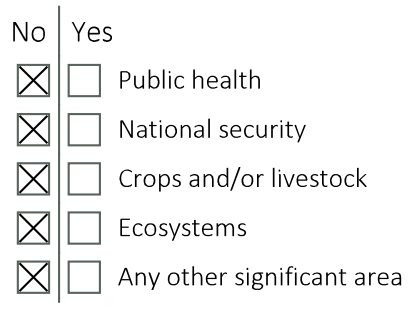

\section{Experiments of concern}

Does the work involve any of these experiments of concern:
No $\mid$ Yes
Х $\square$ Demonstrate how to render a vaccine ineffective
$\bigotimes \square$ Confer resistance to therapeutically useful antibiotics or antiviral agents
$\bigotimes \square$ Enhance the virulence of a pathogen or render a nonpathogen virulent
\ $\square$ Increase transmissibility of a pathogen
Х $\square$ Alter the host range of a pathogen
\ $\square$ Enable evasion of diagnostic/detection modalities
\ $\square$ Enable the weaponization of a biological agent or toxin
Х $\square$ Any other potentially harmful combination of experiments and agents

\section{ChIP-seg}

\section{Data deposition}

$\square$ Confirm that both raw and final processed data have been deposited in a public database such as GEO.

$\square$ Confirm that you have deposited or provided access to graph files (e.g. BED files) for the called peaks.

Data access links

May remain private before publication.

Files in database submission

Genome browser session (e.g. UCSC)

\section{Methodology}

Replicates

Sequencing depth
For "Initial submission" or "Revised version" documents, provide reviewer access links. For your "Final submission" document, provide a link to the deposited data.

Provide a list of all files available in the database submission.

Provide a link to an anonymized genome browser session for "Initial submission" and "Revised version" documents only, to enable peer review. Write "no longer applicable" for "Final submission" documents.
Describe the experimental replicates, specifying number, type and replicate agreement.

Describe the sequencing depth for each experiment, providing the total number of reads, uniquely mapped reads, length of reads and 
Antibodies

Describe the antibodies used for the ChIP-seq experiments; as applicable, provide supplier name, catalog number, clone name, and lot number.

Peak calling parameters

Specify the command line program and parameters used for read mapping and peak calling, including the ChIP, control and index files used.

Data quality

Describe the methods used to ensure data quality in full detail, including how many peaks are at FDR 5\% and above 5-fold enrichment.

Software

Describe the software used to collect and analyze the ChIP-seq data. For custom code that has been deposited into a community repository, provide accession details.

\section{Flow Cytometry}

Plots

Confirm that:

$\square$ The axis labels state the marker and fluorochrome used (e.g. CD4-FITC).

$\square$ The axis scales are clearly visible. Include numbers along axes only for bottom left plot of group (a 'group' is an analysis of identical markers).

$\square$ All plots are contour plots with outliers or pseudocolor plots.

$\square$ A numerical value for number of cells or percentage (with statistics) is provided.

\section{Methodology}

Sample preparation

Instrument

Software

Cell population abundance

Gating strategy

Tick this box to confirm that a figure exemplifying the gating strategy is provided in the Supplementary Information.
Describe the sample preparation, detailing the biological source of the cells and any tissue processing steps used.

Identify the instrument used for data collection, specifying make and model number.

Describe the software used to collect and analyze the flow cytometry data. For custom code that has been deposited into a community repository, provide accession details.

Describe the abundance of the relevant cell populations within post-sort fractions, providing details on the purity of the samples and how it was determined.

Describe the gating strategy used for all relevant experiments, specifying the preliminary FSC/SSC gates of the starting cell population, indicating where boundaries between "positive" and "negative" staining cell populations are defined.

\section{Magnetic resonance imaging}

\section{Experimental design}

Design type

Design specifications

Behavioral performance measures

Acquisition

Imaging type(s)

Field strength

Sequence \& imaging parameters

Area of acquisition

Diffusion MRI

Used
Indicate task or resting state; event-related or block design.

Specify the number of blocks, trials or experimental units per session and/or subject, and specify the length of each trial or block (if trials are blocked) and interval between trials.

State number and/or type of variables recorded (e.g. correct button press, response time) and what statistics were used to establish that the subjects were performing the task as expected (e.g. mean, range, and/or standard deviation across subjects). 
Preprocessing

Preprocessing software

Normalization

Normalization template

Noise and artifact removal

Volume censoring

Statistical modeling \& inference

Model type and settings

Specify type (mass univariate, multivariate, RSA, predictive, etc.) and describe essential details of the model at the first and second levels (e.g. fixed, random or mixed effects; drift or auto-correlation).

Effect(s) tested

Define precise effect in terms of the task or stimulus conditions instead of psychological concepts and indicate whether ANOVA or factorial designs were used.

Specify type of analysis:

Whole brain

ROI-based

Both

Statistic type for inference (See Eklund et al. 2016)

Correction

Models \& analysis

\begin{tabular}{l|l}
$\square / a$ & Involved in the study \\
$\square$ & Functional and/or effective connectivity
\end{tabular}

Functional and/or effective connectivity

Report the measures of dependence used and the model details (e.g. Pearson correlation, partial correlation, mutual information).

Graph analysis

Report the dependent variable and connectivity measure, specifying weighted graph or binarized graph, subject-or group-level, and the global and/or node summaries used (e.g. clustering coefficient, efficiency, etc.)

Multivariate modeling and predictive analysis

Specify independent variables, features extraction and dimension reduction, model, training and evaluation metrics. 